\title{
The fundamental role of the retarded potential in the electrodynamics of superluminal sources
}

\author{
Houshang Ardavan \\ University of Cambridge \\ Arzhang Ardavan \\ University of Oxford \\ John Singleton.* Joseph Fasel, and Andrea Schmidt \\ Los Alamos National Laboratory
}




\begin{abstract}
We calculate the gradient of the radiation field generated by a polarization current with a superluminally rotating distribution pattern and show that the absolute value of this gradient increases as $R^{7 / 2}$ with distance $R$, within the sharply focused subbeams that constitute the overall radiation beam from such a source. In addition to supporting the earlier finding that the azimuthal and polar widths of these subbeams become narrower (as $R^{-3}$ and $R^{-1}$, respectively), with distance from the source, this result implies that the boundary contribution to the solution of the wave equation governing the radiation field does not always vanish in the limit where the boundary tends to infinity (as is commonly assumed in textbooks and the published literature). There is a fundamental difference between the classical expression for the retarded potential and the corresponding retarded solution of the wave equation that governs the electromagnetic field: while the boundary contribution to the retarded solution for the potential can always be rendered equal to zero by means of a gauge transformation that preserves the Lorenz condition, the boundary contribution to the retarded solution of the wave equation for the field may be neglected only if it diminishes with distance faster than the contribution of the source density in the far zone. In the case of a rotating superluminal source, however, the boundary term in the retarded solution for the field is by a factor of the order of $R^{1 / 2}$ larger than the source term of this solution, in the limit where the boundary tends to infinity. This result is consistent with the prediction of the retarded potential that the radiation field generated by a rotating superluminal source decays as $R^{-1 / 2}$, instead of $R^{-1}$, and explains why an argument based on the solution of the wave equation governing the field in which the boundary term is neglected (such as that presented by J. H. Hannay) misses the nonspherical decay of the field. Given that the distribution of the radiation field of an accelerated superluminal source in the far zone is not known a priori, to be prescribed as a boundary condition, our analysis establishes that the only way one can calculate the free-space radiation field of such sources is via the retarded solution for the potential. Finally, we discuss the applicability of these findings to pulsar observational data: the more distant a pulsar, the narrower and brighter its giant pulses should be.
\end{abstract}

*Electronic address: jsingle@lanl.gov 


\section{INTRODUCTION}

Moving sources of electromagnetic radiation whose speeds exceed the speed of light in vacuo have already been generated in the laboratory [1-4]. These sources arise from separation of charges: their superluminally moving distribution patterns are created by the coordinated motion of aggregates of subluminally moving particles. A polarization current density is, however, on the same footing as the current density of free charges in the AmpéreMaxwell equation, so that the propagating distribution patterns of such polarization currents radiate, as would any other moving sources of the electromagnetic field [5- 9 ].

We have already shown, by means of an analysis based on the classical expression for the retarded potential [Eq. (6) below], that the radiation field of a superluminally rotating extended source at a given observation point $\mathrm{P}$ arises almost exclusively from those of its volume elements that approach $\mathrm{P}$, along the radiation direction, with the speed of light and zero acceleration at the retarded time [8-10]. These elements comprise a filamentary part of the source whose radial and azimuthal widths become narrower (as $\delta r \sim R_{\mathrm{P}}{ }^{-2}$ and $\delta \varphi \sim R_{\mathrm{P}}^{-3}$, respectively), the larger the distance $R_{\mathrm{P}}$ of the observer from the source, and whose length is of the order of the length scale $l_{z}$ of the source parallel to the axis of rotation [10]. ( $r, \varphi$, and $z$ are the cylindrical polar coordinates of source points.)

Once a source travels faster than its emitted waves, it can make more than one retarded contribution to the field that is observed at any given instant. This multivaluedness of the retarded time [7-10] means that the wave fronts emitted by each of the contributing volume elements of the source possess an envelope, which in this case consists of a two-sheeted, tubelike surface whose sheets meet tangentially along a spiraling cusp curve (see Figs. 1 and 4 of Ref. [10]). For moderate superluminal speeds, the field inside the envelope receives contributions from three distinct values of the retarded time, while the field outside the envelope is influenced by only a single instant of emission time. Coherent superposition of the emitted waves on the envelope (where two of the contributing retarded times coalesce) and on its cusp (where all three of the contributing retarded times coalesce) results in not only a spatial, but also a temporal focusing of the waves: the contributions from emission over an extended period of retarded time reach an observer who is located on the cusp during a significantly shorter period of observation time.

The field of each contributing volume element of the source is strongest, therefore, on 
the cusp of the envelope of wave fronts that it emits. The bundle of cusps generated by the collection of contributing source elements (i.e., by the filamentary part of the source that approaches the observer with the speed of light and zero acceleration) constitute a radiation subbeam whose widths in the polar and azimuthal directions are of the order of $\delta \theta_{\mathrm{P}} \sim R_{\mathrm{P}}^{-1}$ and $\delta \varphi_{\mathrm{P}} \sim R_{\mathrm{P}}^{-3}$, respectively [10]. $\quad\left(R_{\mathrm{P}}, \varphi_{\mathrm{P}}\right.$ and $\theta_{\mathrm{P}}$ are the spherical polar coordinates of the observation point $\mathrm{P}$.) The overall radiation beam generated by the source consists of a (necessarily incoherent [11]) superposition of such subbeams, a beam whose azimuthal width is the same as the azimuthal extent of the source and whose polar width $\arccos \left[c /\left(r_{<} \omega\right)\right] \leq\left|\theta_{\mathrm{P}}-\pi / 2\right| \leq \arccos \left[c /\left(r_{>} \omega\right)\right]$ is determined by the radial extent $1<\hat{r}_{<} \leq \hat{r} \leq \hat{r}_{>}$of the superluminal part of the source [9, 10]. ( $c$ is the speed of light in vacuo, $\omega$ is the angular frequency of rotation of the source, and $\hat{r} \equiv r \omega / c$.)

Since the cusps only represent the loci of points at which the emitted spherical waves interfere constructively (i.e., represent wave packets that are constantly dispersed and reconstructed out of other waves), the subbeams generated by a superluminal source need not be subject to diffraction as are conventional radiation beams. Nevertheless, they have a decreasing angular width only in the polar direction. Their azimuthal width $\delta \varphi_{\mathrm{P}}$ decreases as $R_{\mathrm{P}}{ }^{-3}$ with distance because they receive contributions from an azimuthal extent $\delta \varphi$ of the source that likewise shrinks as $R_{\mathrm{P}}{ }^{-3}$. They would have had a constant azimuthal width had the azimuthal extent of the contributing part of the source been independent of $R_{\mathrm{P}}$. On the other hand, the solid angle occupied by the cusps has a thickness $\delta z_{\mathrm{P}}$ in the direction parallel to the rotation axis that remains of the order of the height $l_{z}$ of the source distribution at all distances (see Fig. 2 of Ref. [10]). Consequently, the polar width $\delta \theta_{\mathrm{P}}$ of the particular subbeam that goes through the observation point decreases as $R_{\mathrm{P}}{ }^{-1}$, instead of being independent of $R_{\mathrm{P}}[10]$.

Because it is of a constant linear width, parallel to the rotation axis, an individual subbeam subtends an area of the order of $R_{\mathrm{P}}$, rather than $R_{\mathrm{P}}{ }^{2}$. In order that the flux of energy remain the same across all cross sections of the subbeam, therefore, it is essential that the Poynting vector associated with this radiation correspondingly decay more slowly than that of a conventional, spherically decaying radiation: as $R_{\mathrm{P}}{ }^{-1}$, rather than $R_{\mathrm{P}}{ }^{-2}$, within the bundle of cusps that emanate from the constituent volume elements of the source and extend into the far zone. This result, which also follows from the superposition of the Liénard-Wiechert fields of the constituent volume elements of a rotating superluminal source 
[9, 10], has now been demonstrated experimentally [2].

The narrowing of the individual subbeams with distance suggests that the absolute value of the gradient of the radiation field described here should increase with distance, in contrast to that of a conventional, diffracting radiation beam that decreases with distance. This is illustrated by a simple example. Imagine a rotating radiation beam with the amplitude

$$
A\left(R_{\mathrm{P}}, \varphi_{\mathrm{P}}, t_{\mathrm{P}}\right)=A_{0} \hat{R}_{\mathrm{P}}^{1 / 2} \exp \left[-\left(\hat{R}_{\mathrm{P}}^{3} \hat{\varphi}_{\mathrm{P}}\right)^{2}\right]
$$

where $\hat{R}_{\mathrm{P}}$ stands for the scaled distance $R_{\mathrm{P}} \omega / c, \hat{\varphi}_{\mathrm{P}} \equiv \varphi_{\mathrm{P}}-\omega t_{\mathrm{P}}$ is the azimuthal angle in the rotating frame, $t_{\mathrm{P}}$ is the observation time, and $A_{0}$ is a constant. This beam would be observed as a Gaussian pulse that has an azimuthal width of the order of $\hat{R}_{\mathrm{P}}^{-3}$ and carries a constant flux of energy,

$$
\int A^{2} R_{\mathrm{P}}^{2} \sin \theta_{\mathrm{P}} \mathrm{d} \theta_{\mathrm{P}} \mathrm{d} \varphi_{\mathrm{P}}=(2 \pi)^{1 / 2}(c / \omega)^{2} A_{0}^{2}
$$

across any large sphere of radius $R_{\mathrm{P}}$. The gradient of the amplitude of this pulse,

$$
\partial A / \partial \hat{\varphi}_{\mathrm{P}}=-2 A_{0} \hat{R}_{\mathrm{P}}^{7 / 2}\left(\hat{R}_{\mathrm{P}}^{3} \hat{\varphi}_{\mathrm{P}}\right) \exp \left[-\left(\hat{R}_{\mathrm{P}}^{3} \hat{\varphi}_{\mathrm{P}}\right)^{2}\right]
$$

increases in magnitude with distance as $R_{\mathrm{P}}{ }^{7 / 2}$ at the edges of the pulse.

In this paper, we derive the azimuthal (equivalently, temporal) gradient $\left(\partial / \partial \hat{\varphi}_{\mathrm{P}}\right)$ of the radiation field that is generated by a physically viable, rotating superluminal source directly from the retarded potential, and show that the absolute value of this gradient does increase as $\hat{R}_{\mathrm{P}}^{7 / 2}$ within each subbeam. The spiky structure of the angular distribution of the emission from an accelerated superluminal source therefore follows not only from the geometry of the emitted cusps (geometrical optics) that was considered in Ref. [10], but also from the calculation of the field distribution (physical optics) that is presented here. This result corroborates the earlier finding that the overall radiation beam consists of an incoherent superposition of sharply peaked subbeams that become narrower with distance from the source [11].

There is, however, another, more significant implication. The retarded solution to the wave equation that governs the electromagnetic potential in the Lorenz gauge [Eq. (2) below] generally entails three terms: an integral over the retarded value of the electric current density, an integral over the boundary values of the potential and its gradient, and an integral over the initial values of the potential and its time derivative [see Eq. (3) below]. 
For a localized distribution of electric current, the integral over the retarded value of the source density is of the order of $\hat{R}_{\mathrm{P}}^{-1}$ in the far zone. If evaluated for a potential that is of this order of magnitude in the far zone (i.e., decays as $\hat{R}_{\mathrm{P}}^{-1}$ ), the integral over the boundary in this solution would also be of the order of $\hat{R}_{\mathrm{P}}^{-1}$ in the limit where the boundary tends to infinity. However, even potentials that satisfy the Lorenz condition are arbitrary to within a solution of the homogeneous wave equation, so that one can always use the gauge freedom in the choice of potential to set this boundary term identically equal to zero.

In the case of the corresponding retarded solution of the wave equation for the electromagnetic field [Eq. (17) below], on the other hand, one no longer has the freedom offered by a gauge transformation to render the boundary term equal to zero. Nor does this term always decay faster than the source term, so that it could be neglected for a boundary that tends to infinity, as is commonly assumed in textbooks (e.g., page 246 of [12]) and the published literature [13 16]. The boundary contribution to the retarded solution of the wave equation governing the field entails a surface integral over the boundary values of both the field and its gradient [see Eq. (8) below]. In the superluminal regime, where the gradient of the field increases as $\hat{R}_{\mathrm{P}}^{7 / 2}$ over a solid angle that decreases as $R_{\mathrm{P}}{ }^{-4}$, this boundary contribution turns out to be of the order of $\hat{R}_{\mathrm{P}}^{-1 / 2}$ (see Section [V). Not only is this not negligible relative to the contribution from the source term, which decays as $\hat{R}_{\mathrm{P}}^{-1}[13-16]$, but the boundary term constitutes the dominant contribution toward the value of the radiation field in this case.

Thus, if one ignores the boundary term in the retarded solution of the wave equation governing the field (as is done by Hannay [13 16]), one would obtain a result, in the superluminal regime, that contradicts what is obtained by calculating the field via the retarded potential [8 -10]. However, the contradiction stems solely from having ignored a term in the solution to the wave equation that is by a factor of the order of $\hat{R}_{\mathrm{P}}^{1 / 2}$ greater than the term that is normally kept in this solution. The contradiction disappears once the neglected term is taken into account: the solutions to both the wave equation that governs the potential and the wave equation that governs the field predict that the field of a rotating superluminal source decays as $\hat{R}_{\mathrm{P}}^{-1 / 2}$ as $R_{\mathrm{P}}$ tends to infinity.

From a physical point of view, however, what one obtains by including the boundary term in the retarded solution to the wave equation that governs the field is merely a mathematical identity; it is not a solution that could be used to calculate the field arising from a given source distribution in free space. Unless its boundary term happens to be negligibly smaller 
than its source term, a condition that cannot be known a priori, the solution in question would require that one prescribe the field in the radiation zone (i.e., what one is seeking) as a boundary condition. The role played by the classical expression for the retarded potential in radiation theory is clearly much more fundamental than that played by the corresponding retarded solution of the wave equation governing the field. The only way to calculate the free-space radiation field of an accelerated superluminal source is to calculate the retarded potential and differentiate the resulting expression to find the field (see also [17]). We must again emphasize that this is an important contrast with subluminal sources.

This paper is organized as follows: Section III presents the retarded solutions to the initial-boundary value problems for the wave equations that govern the potential and the field. We provide a detailed mathematical derivation of the gradient of the radiation field that is generated by a rotating superluminal source in Section III, with a brief account of the required background material in Subsection IIIA, the formulation of the problem in Subsection IIIB, the derivation of an integral representation of the gradient of the Green's function in Subsection \IC, the regularization of the integral over the radial extent of the source in Subsection IIID (and Appendix A), a description of contours of steepest descent in Subsection IIIE, and the asymptotic evaluation of the gradient of the radiation field in Subsection [IIF. Section IV evaluates the boundary term in the retarded solution to the wave equation governing the field, and we conclude in Section V.

\section{BOUNDARY TERM IN THE SOLUTION TO THE WAVE EQUATION}

In the Lorenz gauge, the electromagnetic fields

$$
\mathbf{E}=-\nabla_{\mathrm{P}} A^{0}-\partial \mathbf{A} / \partial\left(c t_{\mathrm{P}}\right), \quad \mathbf{B}=\nabla_{\mathrm{P}} \times \mathbf{A}
$$

are given by a four-potential $A^{\mu}$ that satisfies the wave equation

$$
\nabla^{2} A^{\mu}-\frac{1}{c^{2}} \frac{\partial^{2} A^{\mu}}{\partial t^{2}}=-\frac{4 \pi}{c} j^{\mu}, \quad \mu=0, \cdots, 3,
$$

where $A^{0} / c$ and $j^{0} / c$ are the electric potential and the charge density, and $A^{\mu}$ and $j^{\mu}$ for $\mu=1,2,3$ are the components of the magnetic potential $\mathbf{A}$ and the current density $\mathbf{j}$ in a

Cartesian coordinate system [12]. The solution to the initial-boundary value problem for 
Eq. (22) is given by

$$
\begin{aligned}
A^{\mu}\left(\mathbf{x}_{\mathrm{P}}, t_{\mathrm{P}}\right)= & \frac{1}{c} \int_{0}^{t_{\mathrm{P}}} \mathrm{d} t \int_{V} \mathrm{~d}^{3} x j^{\mu} G+\frac{1}{4 \pi} \int_{0}^{t_{\mathrm{P}}} \mathrm{d} t \int_{\Sigma} \mathrm{d} \mathbf{S} \cdot\left(G \nabla A^{\mu}-A^{\mu} \nabla G\right) \\
& -\frac{1}{4 \pi c^{2}} \int_{V} \mathrm{~d}^{3} x\left(A^{\mu} \frac{\partial G}{\partial t}-G \frac{\partial A^{\mu}}{\partial t}\right)_{t=0},
\end{aligned}
$$

in which $G$ is the Green's function and $\Sigma$ is the surface enclosing the volume $V$ (see, e.g., page 893 of [18]).

The potential that arises from a time-dependent localized source in unbounded space decays as $R_{\mathrm{P}}{ }^{-1}$ when $R_{\mathrm{P}} \gg 1$, so that for an arbitrary free-space potential the second term in Eq. (3) would be of the same order of magnitude $\left(\sim R_{\mathrm{P}}{ }^{-1}\right)$ as the first term in the limit that the boundary $\Sigma$ tends to infinity. However, even potentials that satisfy the Lorenz condition $\nabla \cdot \mathbf{A}+c^{-2} \partial A^{0} / \partial t=0$ are arbitrary to within a solution of the homogeneous wave equation: the gauge transformation

$$
\mathbf{A} \rightarrow \mathbf{A}+\nabla \Lambda, \quad A^{0} \rightarrow A^{0}-\partial \Lambda / \partial t
$$

preserves the Lorenz condition if $\nabla^{2} \Lambda-c^{-2} \partial^{2} \Lambda / \partial t^{2}=0$ (see [12]). One can always use this gauge freedom in the choice of the potential to render the boundary contribution (the second term) in Eq. (3) equal to zero, since this term, too, satisfies the homogenous wave equation. Under the null initial conditions $\left.A^{\mu}\right|_{t=0}=\left(\partial A^{\mu} / \partial t\right)_{t=0}=0$, assumed in this paper, the contribution from the third term in Eq. (3) is identically zero.

In the absence of boundaries, the retarded Green's function has the form

$$
G\left(\mathbf{x}, t ; \mathbf{x}_{\mathrm{P}}, t_{\mathrm{P}}\right)=\frac{\delta\left(t_{\mathrm{P}}-t-R / c\right)}{R},
$$

where $\delta$ is the Dirac delta function and $R$ is the magnitude of the separation $\mathbf{R} \equiv \mathbf{x}_{\mathrm{P}}-\mathbf{x}$ between the observation point $\mathbf{x}_{\mathrm{P}}$ and the source point $\mathbf{x}$. Irrespective of whether the radiation decays spherically or nonspherically, therefore, the potential $A^{\mu}$ due to a localized source distribution, which is switched on at $t=0$ in an unbounded space, can be calculated from the first term in Eq. (3) :

$$
A^{\mu}\left(\mathbf{x}_{\mathrm{P}}, t_{\mathrm{P}}\right)=c^{-1} \int \mathrm{d}^{3} x \mathrm{~d} t j^{\mu}(\mathbf{x}, t) \delta\left(t_{\mathrm{P}}-t-R / c\right) / R,
$$

i.e., from the classical expression for the retarded potential. Whatever the Green's function for the problem may be in the presence of boundaries, it would approach that in Eq. (5) in 
the limit where the boundaries tend to infinity, so that one can also use this potential to calculate the field on a boundary that lies at large distances from the source.

Next, let us consider the wave equation that governs the magnetic field

$$
\nabla^{2} \mathbf{B}-\frac{1}{c^{2}} \frac{\partial^{2} \mathbf{B}}{\partial t^{2}}=-\frac{4 \pi}{c} \nabla \times \mathbf{j}
$$

This may be obtained by simply taking the curl of the wave equation for the vector potenial [Eq. (2) for $\mu=1,2,3]$. We write the solution to the initial-boundary value problem for Eq. (17), in analogy with Eq. (3), as

$$
\begin{aligned}
B_{k}\left(\mathbf{x}_{\mathrm{P}}, t_{\mathrm{P}}\right)= & \frac{1}{c} \int_{0}^{t_{\mathrm{P}}} \mathrm{d} t \int_{V} \mathrm{~d}^{3} x(\nabla \times \mathbf{j})_{k} G+\frac{1}{4 \pi} \int_{0}^{t_{\mathrm{P}}} \mathrm{d} t \int_{\Sigma} \mathrm{d} \mathbf{S} \cdot\left(G \nabla B_{k}-B_{k} \nabla G\right) \\
& -\frac{1}{4 \pi c^{2}} \int_{V} \mathrm{~d}^{3} x\left(B_{k} \frac{\partial G}{\partial t}-G \frac{\partial B_{k}}{\partial t}\right)_{t=0},
\end{aligned}
$$

where $k=1,2,3$ designate the components of $\mathbf{B}$ and $\nabla \times \mathbf{j}$ in a Cartesian coordinate system. Here, we no longer have the freedom, offered in the case of Eq. (3) by a gauge transformation, to make the boundary term zero.

Our task in this paper is to demonstrate that the boundary contribution in Eq. (8) is, in fact, by a factor of the order of $\hat{R}_{\mathrm{P}}^{1 / 2}$ larger than the source term of this equation in the far zone when the source is superluminal and accelerated. For this purpose, we need to know how the the gradient $\nabla B_{k}$ in the second term in Eq. (8) decays in the far zone. We shall calculate, in the following section, the field $\mathbf{B}$ and its gradient directly from the classical expression for the retarded potential [Eq. (6) ], and use the resulting expressions to evaluate the second term in Eq. (8) for a boundary that lies in the far zone.

\section{GRADIENT OF THE RADIATION FIELD GENERATED BY A ROTATING SUPERLUMINAL SOURCE}

\section{A. Background: The exact expression for the radiation field}

We base our analysis on the generic superluminal source distribution considered in

Refs. [9] and [10], which has already been created in the laboratory [2]. This source comprises a polarization current density $\mathbf{j}=\partial \mathbf{P} / \partial t$ for which

$$
P_{r, \varphi, z}(r, \varphi, z, t)=s_{r, \varphi, z}(r, z) \cos (m \hat{\varphi}) \cos (\Omega t), \quad-\pi<\hat{\varphi} \leq \pi
$$


with

$$
\hat{\varphi} \equiv \varphi-\omega t
$$

where $P_{r, \varphi, z}$ are the components of the polarization $\mathbf{P}$ in a cylindrical coordinate system based on the axis of rotation, $\mathbf{s}(r, z)$ is an arbitrary vector that vanishes outside a finite region of the $(r, z)$ space, and $m$ is a positive integer. For a fixed value of $t$, the azimuthal dependence of the density (8) along each circle of radius $r$ within the source is the same as that of a sinusoidal wave train, of wavelength $2 \pi r / m$ whose $m$ cycles fit around the circumference of the circle smoothly. As time elapses, this wave train both propagates around each circle with the velocity $r \omega$ and oscillates in its amplitude with the frequency $\Omega$. This is a generic source: one can construct any distribution with a uniformly rotating pattern, $P_{r, \varphi, z}(r, \hat{\varphi}, z)$, by the superposition over $m$ of terms of the form $s_{r, \varphi, z}(r, z, m) \cos (m \hat{\varphi})$.

To find the retarded field that follows from Eq. (66) for the source described in Eq. (9), we first calculated in Ref. [9] the Liénard-Wiechert field of a circularly moving point source with a speed $r \omega>c$, i.e., a generalization of the synchrotron radiation to the superluminal regime. We then evaluated the integral representing the retarded field (rather than the retarded potential) of the extended source (8) by superposing the fields generated by the constituent volume elements of this source, i.e., by using the generalization of the synchrotron field as the Green's function for the problem (see also [17]). In the superluminal regime, this Green's function has extended singularities that arise from the coherent superposition of the emitted waves on the envelope of wave fronts and its cusp.

Inserting the expression for $\mathbf{j}=\partial \mathbf{P} / \partial t$ from Eq. (9) into Eq. (66), and changing the variables of integration from $(\mathbf{x}, t)=(r, \varphi, z, t)$ to $(r, \varphi, z, \hat{\varphi})$, we found in Eq. (20) of Ref. [9] that the magnetic field $\mathbf{B}$ of the generated radiation is given by

$$
\mathbf{B}=-\frac{1}{2} \mathrm{i}(\omega / c)^{2} \sum_{\mu=\mu_{ \pm}} \int_{V} r \mathrm{~d} r \mathrm{~d} \hat{\varphi} \mathrm{d} z \mu \exp (-\mathrm{i} \mu \hat{\varphi}) \sum_{j=1}^{3} \mathbf{u}_{j} \partial G_{j} / \partial \hat{\varphi},
$$

where $\mu_{ \pm} \equiv(\Omega / \omega) \pm m$,

$$
\mathbf{u}_{1} \equiv s_{r} \cos \theta_{\mathrm{P}} \hat{\mathbf{e}}_{\|}+s_{\varphi} \hat{\mathbf{e}}_{\perp}, \quad \mathbf{u}_{2} \equiv-s_{\varphi} \cos \theta_{\mathrm{P}} \hat{\mathbf{e}}_{\|}+s_{r} \hat{\mathbf{e}}_{\perp}, \quad \mathbf{u}_{3} \equiv-s_{z} \sin \theta_{\mathrm{P}} \hat{\mathbf{e}}_{\|},
$$

and $G_{j}(j=1,2,3)$ are the functions resulting from the remaining integration with respect to $\varphi$ :

$$
\left[\begin{array}{l}
G_{1} \\
G_{2} \\
G_{3}
\end{array}\right]=\int_{\Delta \varphi} \mathrm{d} \varphi \frac{\delta(g-\phi)}{R} \exp (\mathrm{i} \Omega \varphi / \omega)\left[\begin{array}{c}
\cos \left(\varphi-\varphi_{\mathrm{P}}\right) \\
\sin \left(\varphi-\varphi_{\mathrm{P}}\right) \\
1
\end{array}\right]
$$


Here $\phi$ stands for $\hat{\varphi}-\hat{\varphi}_{\mathrm{P}}$ with $\hat{\varphi}_{\mathrm{P}} \equiv \varphi_{\mathrm{P}}-\omega t_{\mathrm{P}}, R$ is

$$
R=\left[\left(z_{\mathrm{P}}-z\right)^{2}+r_{\mathrm{P}}^{2}+r^{2}-2 r_{\mathrm{P}} r \cos \left(\varphi_{\mathrm{P}}-\varphi\right)\right]^{\frac{1}{2}},
$$

the function $g$ is defined by

$$
g \equiv \varphi-\varphi_{\mathrm{P}}+\hat{R}
$$

with $\hat{R} \equiv R \omega / c, \Delta \varphi$ is the interval of azimuthal angle traversed by the source, and $V$ is the volume occupied by the source in the $(r, \hat{\varphi}, z)$ space. The unit vector $\hat{\mathbf{e}}_{\|} \equiv\left(\hat{\mathbf{e}}_{z} \times \hat{\mathbf{n}}\right) /\left|\hat{\mathbf{e}}_{z} \times \hat{\mathbf{n}}\right|$ (which is parallel to the plane of rotation), $\hat{\mathbf{e}}_{\perp} \equiv \hat{\mathbf{n}} \times \hat{\mathbf{e}}_{\|}$, and the radiation direction $\hat{\mathbf{n}} \equiv \mathbf{R} / R$ together form an orthonormal triad ( $\hat{\mathbf{e}}_{z}$ is the base vector associated with the coordinate $z)$. The corresponding expression for the electric field in the limit $R_{\mathrm{P}} \equiv\left|\mathbf{x}_{\mathrm{P}}\right| \rightarrow \infty$, where $\hat{\mathbf{n}} \simeq \mathbf{x}_{\mathrm{P}} /\left|\mathbf{x}_{\mathrm{P}}\right|$, is given by $\mathbf{E}=\hat{\mathbf{n}} \times \mathbf{B}$, as in any other radiation.

A distinctive feature of the emission from a superluminal source is the multivaludeness of the retarded time [7-10]. At any given observation time, at least three distinct contributions, arising from three differing retarded times, are made toward the value of the radiation field by the part of the source that lies within the following volume of the $(r, \hat{\varphi}, z)$ space:

$$
\Delta \geq 0, \quad \phi_{-} \leq \phi \leq \phi_{+},
$$

where

$$
\begin{gathered}
\Delta=\left(\hat{r}_{\mathrm{P}}^{2}-1\right)\left(\hat{r}^{2}-1\right)-\left(\hat{z}-\hat{z}_{\mathrm{P}}\right)^{2}, \\
\phi_{ \pm}=2 \pi-\arccos \left[\left(1 \mp \Delta^{\frac{1}{2}}\right) /\left(\hat{r} \hat{r}_{\mathrm{P}}\right)\right]+\hat{R}_{ \pm},
\end{gathered}
$$

and

$$
\hat{R}_{ \pm}=\left[\left(\hat{z}-\hat{z}_{\mathrm{P}}\right)^{2}+\hat{r}^{2}+\hat{r}_{\mathrm{P}}^{2}-2\left(1 \mp \Delta^{\frac{1}{2}}\right)\right]^{\frac{1}{2}}
$$

This volume is bounded by a two-sheeted surface, the so-called bifurcation surface, whose two sheets $\phi=\phi_{ \pm}(r, z)$ meet tangentially along a cusp (see Figs. 3 and 4 of Ref. [9]). The strongest contributions are made by the source elements that lie close to the cusp curve $\Delta=0, \phi=\left.\phi_{ \pm}\right|_{\Delta=0}$, where the two sheets of the bifurcation surface meet tangentially. For $\hat{R}_{\mathrm{P}} \gg 1$, the filamentary locus of these contributing source elements is essentially parallel to the rotation axis and has exceedingly narrow radial and azimuthal widths, of the orders of $\hat{R}_{\mathrm{P}}^{-2}$ and $\hat{R}_{\mathrm{P}}^{-3}$, respectively (see Fig. 2 of Ref. [10]) 
The asymptotic values of the Green's functions $G_{j}$ close to the cusp curve of the bifurcation surface (where $\Delta \ll 1$ ) are given by

$$
G_{j}= \begin{cases}G_{j}^{\text {in }} & |\chi|<1 \\ G_{j}^{\text {out }} & |\chi|>1\end{cases}
$$

with

$$
G_{j}^{\text {in }} \simeq 2 c_{1}^{-2}\left(1-\chi^{2}\right)^{-\frac{1}{2}}\left[p_{j} \cos \left(\frac{1}{3} \arcsin \chi\right)-c_{1} q_{j} \sin \left(\frac{2}{3} \arcsin \chi\right)\right]
$$

and

$$
G_{j}{ }^{\text {out }} \simeq c_{1}^{-2}\left(\chi^{2}-1\right)^{-\frac{1}{2}}\left[p_{j} \sinh \left(\frac{1}{3} \operatorname{arccosh}|\chi|\right)+c_{1} q_{j} \operatorname{sgn}(\chi) \sinh \left(\frac{2}{3} \operatorname{arccosh}|\chi|\right)\right]
$$

where

$$
\chi \equiv 3\left(\phi-c_{2}\right) /\left(2 c_{1}^{3}\right)
$$

with

$$
c_{1} \equiv\left(\frac{3}{4}\right)^{\frac{1}{3}}\left(\phi_{+}-\phi_{-}\right)^{\frac{1}{3}}, \quad c_{2} \equiv \frac{1}{2}\left(\phi_{+}+\phi_{-}\right)
$$

and the symbol $\simeq$ denotes asymptotic approximation. The derivation of these asymptotic values, together with the exact expressions for the coefficients $p_{j}(r, z)$ and $q_{j}(r, z)$ may be found in the Appendices of Refs. [8] and [9]. Here, we only need the following limiting values of these coefficients for $\hat{R}_{\mathrm{P}} \gg 1$ :

$$
\begin{gathered}
p_{1} \simeq 2^{\frac{1}{3}}(\omega / c) \hat{R}_{\mathrm{P}}^{-2} \exp \left(\mathrm{i} \Omega \varphi_{c} / \omega\right), \\
p_{2} \simeq-\hat{R}_{\mathrm{P}} p_{1}, \quad p_{3} \simeq-p_{2},
\end{gathered}
$$

and

$$
\begin{gathered}
q_{1} \simeq 2^{\frac{2}{3}}(\omega / c) \hat{R}_{\mathrm{P}}^{-1} \exp \left(\mathrm{i} \Omega \varphi_{c} / \omega\right), \\
q_{2} \simeq-q_{3} \simeq-\mathrm{i}(\Omega / \omega) q_{1}
\end{gathered}
$$

where $\varphi_{c} \simeq \varphi_{\mathrm{P}}+3 \pi / 2$ in this limit. Note that, in these expressions, $G_{j}^{\text {in,out }}$ represent the different forms assumed by the Green's functions $G_{j}$ inside and outside the bifurcation surface, i.e., for $\phi$ inside and outside the interval $\left(\phi_{-}, \phi_{+}\right)$respectively (see Fig. 6 of Ref. [9]). 
The above results show that as a source point $(r, \hat{\varphi}, z)$ in the vicinity of the cusp curve $\Delta=0, \phi=\left.\phi_{ \pm}\right|_{\Delta=0}$, approaches the bifurcation surface from inside, i.e., as $\chi \rightarrow 1$ - or $\chi \rightarrow-1+, G_{j}{ }^{\text {in }}$ and hence $G_{j}$ diverge. However, as a source point approaches one of the sheets of the bifurcation surface from outside, $G_{j}$ tends to a finite limit:

$$
\left.G_{j}{ }^{\text {out }}\right|_{\phi=\phi_{ \pm}}=\left.G_{j}{ }^{\text {out }}\right|_{\chi= \pm 1} \simeq\left(p_{j} \pm 2 c_{1} q_{j}\right) /\left(3 c_{1}^{2}\right) ;
$$

for, the numerator of $G_{j}{ }^{\text {out }}$ is also zero when $|\chi|=1$. The Green's function $G_{j}$ is singular, in other words, only on the inner side of the bifurcation surface (see Fig. 6 of Ref. [9]).

\section{B. Formulation of the problem}

It turns out that none of the componenets of the gradient of $\mathbf{B}$ can be evaluated for the source distribution (9) without a lengthy calculation. However, we shall see in Section IV that the radial component of $\nabla B_{k}$ is of the same order of magnitude in the far zone as the azimuthal (or equivalently, temporal) component $\partial B_{k} / \partial \hat{\varphi}_{\mathrm{P}}$ of the gradient of $B_{k}\left(r_{\mathrm{P}}, \hat{\varphi}_{\mathrm{P}}, z_{\mathrm{P}}\right)$. Since this component of the field gradient is both algebraically simpler to calculate and more

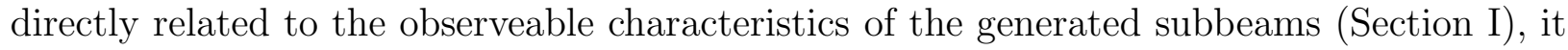
will be the only component that we shall here evaluate in detail. The relationship between the far-field values of this and the other components of the field gradient is not difficult to establish (Section IV).

The component $\partial \mathbf{B} / \partial \hat{\varphi}_{\mathrm{P}}$ of the gradient of $\mathbf{B}$ may be calculated by differentiating the right-hand side of Eq. (11) under the integral sign and using the fact that $\partial G / \partial \hat{\varphi}_{\mathrm{P}}=$ $-\partial G / \partial \hat{\varphi}$. It follows from an argument identical to that given in Ref. [9] (in connection with calculating $\mathbf{B}$ itself $)$ that the contribution $\left(\partial \mathbf{B} / \partial \hat{\varphi}_{\mathrm{P}}\right)_{\Delta \geq 0}$ arising from the source elements in $\Delta \geq 0$ toward the value of $\partial \mathbf{B} / \partial \hat{\varphi}_{\mathrm{P}}$ can be written as

$$
\left(\partial \mathbf{B} / \partial \hat{\varphi}_{\mathrm{P}}\right)_{\Delta \geq 0}=\left(\partial \mathbf{B} / \partial \hat{\varphi}_{\mathrm{P}}\right)^{\text {in }}+\left(\partial \mathbf{B} / \partial \hat{\varphi}_{\mathrm{P}}\right)^{\text {out }}
$$

with

$$
\left(\partial \mathbf{B} / \partial \hat{\varphi}_{\mathrm{P}}\right)^{\mathrm{in}, \text { out }}=\frac{1}{2} \mathrm{i}(\omega / c)^{2} \sum_{j=1}^{3} \int_{\Delta \geq 0} r \mathrm{~d} r \mathrm{~d} z \mathbf{u}_{j} L_{j}{ }^{\text {in,out }},
$$

where

$$
L_{j}^{\text {in }}=\sum_{\mu=\mu_{ \pm}} \int_{\phi_{-}}^{\phi_{+}} \mathrm{d} \phi \mu \exp (-\mathrm{i} \mu \hat{\varphi})\left(\partial^{2} G_{j} / \partial \hat{\varphi}^{2}\right)^{\mathrm{in}}
$$


and

$$
L_{j}{ }^{\text {out }}=\sum_{\mu=\mu_{ \pm}}\left(\int_{-\pi-\hat{\varphi}_{\mathrm{P}}}^{\phi_{-}}+\int_{\phi_{+}}^{\pi-\hat{\varphi}_{\mathrm{P}}}\right) \mathrm{d} \phi \mu \exp (-\mathrm{i} \mu \hat{\varphi})\left(\partial^{2} G_{j} / \partial \hat{\varphi}^{2}\right)^{\text {out }} .
$$

Once it is integrated by parts, the integral in Eq. (29b) in turn splits into three terms:

$$
\begin{aligned}
L_{j}^{\mathrm{in}}= & \sum_{\mu=\mu_{ \pm}}\left\{\left.\mu \exp (-\mathrm{i} \mu \hat{\varphi})\left[\left(\partial G_{j} / \partial \hat{\varphi}\right)^{\mathrm{in}}+\mathrm{i} \mu G_{j}{ }^{\mathrm{in}}\right]\right|_{\phi_{-}} ^{\phi_{+}}\right. \\
& \left.-\mu^{3} \int_{\phi_{-}}^{\phi_{+}} \mathrm{d} \phi \exp (-\mathrm{i} \mu \hat{\varphi}) G_{j}{ }^{\mathrm{in}}\right\},
\end{aligned}
$$

of which the first two (integrated) terms are divergent [see Eq. (20b)]. Hadamard's finite part of $L_{j}{ }^{\text {in }}$ and hence of $\left(\partial \mathbf{B} / \partial \hat{\varphi}_{\mathrm{P}}\right)^{\text {in }}$, here designated by the prefix $\mathcal{F}$, is obtained by discarding this divergent contribution toward the value of $L_{j}{ }^{\text {in }}$ (see Refs. [9] and [19]:

$$
\mathcal{F}\left\{L_{j}{ }^{\mathrm{in}}\right\}=-\mu^{3} \sum_{\mu=\mu_{ \pm}} \int_{\phi_{-}}^{\phi_{+}} \mathrm{d} \phi \exp (-\mathrm{i} \mu \hat{\varphi}) G_{j}{ }^{\mathrm{in}} .
$$

Note that the singularity of the kernel of this integral, i.e., the singularity of $G_{j}{ }^{\text {in }}$, is like that of $\left|\hat{\varphi}_{ \pm}-\hat{\varphi}\right|^{-\frac{1}{2}}$ and so is integrable.

The boundary contributions from $\phi=\phi_{ \pm}$that result from the integration of the righthand side of Eq. (29c) by parts are well-defined automatically:

$$
\begin{aligned}
L_{j}{ }^{\text {out }}= & -\sum_{\mu=\mu_{ \pm}}\left\{\left.\mu \exp (-\mathrm{i} \mu \hat{\varphi})\left[\left(\partial G_{j} / \partial \hat{\varphi}\right)^{\text {out }}+\mathrm{i} \mu G_{j}{ }^{\text {out }}\right]\right|_{\phi_{-}} ^{\phi_{+}}\right. \\
& \left.+\left(\int_{-\pi-\hat{\varphi}_{\mathrm{P}}}^{\phi_{-}}+\int_{\phi_{+}}^{\pi-\hat{\varphi}_{\mathrm{P}}}\right) \mathrm{d} \phi \mu^{3} \exp (-\mathrm{i} \mu \hat{\varphi}) G_{j}{ }^{\text {out }}\right\},
\end{aligned}
$$

since $\left(\partial G_{j} / \partial \hat{\varphi}\right)^{\text {out }}$ (like $\left.G_{j}{ }^{\text {out }}\right)$ tends to a finite limit as the bifurcation surface is approached from outside (see Subsection IIIC). In deriving Eq. (32), we have made use of the fact that $\left.\left(\partial G_{j} / \partial \hat{\varphi}\right)^{\text {out }}\right|_{\phi=\pi-\hat{\varphi}_{\mathrm{P}}}$ equals $\left.\left(\partial G_{j} / \partial \hat{\varphi}\right)^{\text {out }}\right|_{\phi=-\pi-\hat{\varphi}_{\mathrm{P}}}$ when $\phi_{ \pm} \neq \pm \pi-\hat{\varphi}_{\mathrm{P}}$. The integral representing $L_{j}{ }^{\text {out }}$, in other words, is finite by itself and needs no regularization.

If we now insert $\mathcal{F}\left\{L_{j}{ }^{\text {in }}\right\}$ and $L_{j}{ }^{\text {out }}$ from Eqs. (31) and (32) in Eq. (29a) and combine $\left(\partial \mathbf{B} / \partial \hat{\varphi}_{\mathrm{P}}\right)^{\text {in }}$ and $\left(\partial \mathbf{B} / \partial \hat{\varphi}_{\mathrm{P}}\right)^{\text {out }}$, we arrive at an expression for the Hadamard finite part of $\left(\partial \mathbf{B} / \partial \hat{\varphi}_{\mathrm{P}}\right)_{\Delta \geq 0}$ which entails both a volume and a surface integral:

$$
\mathcal{F}\left\{\left(\partial \mathbf{B} / \partial \hat{\varphi}_{\mathrm{P}}\right)_{\Delta \geq 0}\right\}=\left(\partial \mathbf{B} / \partial \hat{\varphi}_{\mathrm{P}}\right)^{\mathrm{s}}+\left(\partial \mathbf{B} / \partial \hat{\varphi}_{\mathrm{P}}\right)^{\mathrm{ns}} .
$$

The volume integral

$$
\begin{aligned}
\left(\partial \mathbf{B} / \partial \hat{\varphi}_{\mathrm{P}}\right)^{\mathrm{s}}= & -\frac{1}{2} \mathrm{i}(\omega / c)^{2} \sum_{\mu=\mu_{ \pm}} \mu^{3} \int_{\Delta \geq 0} r \mathrm{~d} r \mathrm{~d} z \int_{-\pi}^{\pi} \mathrm{d} \hat{\varphi} \exp (-\mathrm{i} \mu \hat{\varphi}) \\
& \times \sum_{j=i}^{3} \mathbf{u}_{j} G_{j}
\end{aligned}
$$


has the same form as the familiar integral representation of the field of a subluminal source [12] and decays spherically (as $R_{\mathrm{P}}^{-1}$ for $\hat{R}_{\mathrm{P}} \gg 1$ ).

The surface integral

$$
\left(\partial \mathbf{B} / \partial \hat{\varphi}_{\mathrm{P}}\right)^{\mathrm{ns}} \equiv-\frac{1}{2} \mathrm{i}(\omega / c)^{2} \sum_{j=1}^{3} \int_{\Delta \geq 0} r \mathrm{~d} r \mathrm{~d} z \mathbf{u}_{j} L_{j}{ }^{\text {edge }}
$$

stems from the boundary contribution

$$
\left.L_{j}^{\text {edge }} \equiv \sum_{\mu=\mu_{ \pm}} \mu \exp (-\mathrm{i} \mu \hat{\varphi})\left[\left(\partial G_{j} / \partial \hat{\varphi}\right)^{\text {out }}+\mathrm{i} \mu G_{j}{ }^{\text {out }}\right]\right|_{\phi_{-}} ^{\phi_{+}}
$$

in Eq. (32). It is this contribution that turns out to increase, rather than decay, in the limit $R_{\mathrm{P}} \rightarrow \infty$. To see this, we need to know the values of $\left(\partial G_{j} / \partial \hat{\varphi}\right)^{\text {out }}$ at $\phi=\phi_{ \pm}$, in addition to those of $\left.G_{j}{ }^{\text {out }}\right|_{\phi=\phi_{ \pm}}$which are given in Eq. (27).

\section{Azimuthal (or temporal) gradient of the Green's function}

The Green's function (13) depends on $\hat{\varphi}$ only through the variable $\phi$ which appears in the argument of the Dirac delta function, so that the differentiation of Eq. (13) with respect to $\hat{\varphi}$ simply yields

$$
\partial G_{j} / \partial \hat{\varphi}=-\int_{\Delta \varphi} \mathrm{d} \varphi h_{j}(\varphi) \delta^{\prime}(g-\phi),
$$

where $\delta^{\prime}$ stands for the derivative of the delta function with respect to its argument, and

$$
\left[\begin{array}{l}
h_{1} \\
h_{2} \\
h_{3}
\end{array}\right]=\frac{\exp (\mathrm{i} \Omega \varphi / \omega)}{R}\left[\begin{array}{c}
\cos \left(\varphi-\varphi_{\mathrm{P}}\right) \\
\sin \left(\varphi-\varphi_{\mathrm{P}}\right) \\
1
\end{array}\right] \text {. }
$$

Integrating the right-hand side of Eq. (37) by parts, we obtain

$$
\begin{aligned}
\frac{\partial G_{j}}{\partial \hat{\varphi}} & =-\int_{\Delta \varphi} \mathrm{d} \varphi \frac{h_{j}(\varphi)}{\partial g / \partial \varphi} \frac{\mathrm{d}}{\mathrm{d} \varphi} \delta(g-\phi) \\
& =\int_{\Delta \varphi} \mathrm{d} \varphi \frac{\mathrm{d}}{\mathrm{d} \varphi}\left[\frac{h_{j}(\varphi)}{\partial g / \partial \varphi}\right] \delta(g-\phi),
\end{aligned}
$$

when the source trajectory intersects the bifurcation surface of the observation point (i.e., the argument of the delta function vanishes within $\Delta \varphi$ ). A uniform asymptotic approximation to this integral, for small $\Delta$, can be found by the method of Chester et al. in the time domain [20, 21]. 
Where it is analytic (i.e., for all $\mathbf{x} \neq \mathbf{x}_{\mathrm{P}}$ ), the function $g(\varphi)$ transforms to the cubic function

$$
g(\varphi)=\frac{1}{3} \nu^{3}-c_{1}^{2} \nu+c_{2}
$$

where $\nu$ is a new variable of integration replacing $\varphi$ and the coefficients $c_{1}$ and $c_{2}$ [defined in Eq. (22)] are such that the values of the two functions on opposite sides of Eq. (40) coincide at their extrema. Insertion of Eq. (40) and its derivative,

$$
\frac{\partial g}{\partial \varphi}=\frac{\nu^{2}-c_{1}^{2}}{\mathrm{~d} \varphi / \mathrm{d} \nu}
$$

in Eq. (39) results in

$$
\frac{\partial G_{j}}{\partial \hat{\varphi}}=\int_{\Delta \nu} \mathrm{d} \nu\left[-\frac{F_{j}}{\left(\nu^{2}-c_{1}^{2}\right)^{2}}+\frac{F^{\prime}{ }_{j}}{\nu^{2}-c_{1}{ }^{2}}\right] \delta\left(\frac{1}{3} \nu^{3}-c_{1}{ }^{2} \nu+c_{2}-\phi\right),
$$

where

$$
\begin{gathered}
F_{j} \equiv\left(\frac{\mathrm{d} \varphi}{\mathrm{d} \nu}\right)^{3} \frac{\partial^{2} g}{\partial \varphi^{2}} h_{j}, \\
F^{\prime}{ }_{j} \equiv\left(\frac{\mathrm{d} \varphi}{\mathrm{d} \nu}\right)^{2} \frac{\partial h_{j}}{\partial \varphi},
\end{gathered}
$$

and $\Delta \nu$ is the image of $\Delta \varphi$ under transformation (40).

As in the evaluation of $G_{j}$ in Refs. [8] and [9], the leading term in the asymptotic expansion of the integral (42a) for small $c_{1}$, which corresponds to small $\Delta$ [see Eq. (53) below], can now be obtained by replacing the functions $F$ and $F^{\prime}$ in its integrand with $P_{j}+Q_{j} \nu$ and $P^{\prime}{ }_{j}+Q^{\prime}{ }_{j} \nu$, respectively, and extending its range $\Delta \nu$ to $(-\infty, \infty)$ :

$$
\frac{\partial G_{j}}{\partial \hat{\varphi}} \simeq \int_{-\infty}^{\infty} \mathrm{d} \nu\left[-\frac{P_{j}+Q_{j} \nu}{\left(\nu^{2}-c_{1}{ }^{2}\right)^{2}}+\frac{P^{\prime}{ }_{j}+Q^{\prime}{ }_{j} \nu}{\nu^{2}-c_{1}{ }^{2}}\right] \delta\left(\frac{1}{3} \nu^{3}-c_{1}{ }^{2} \nu+c_{2}-\phi\right),
$$

where

$$
\begin{gathered}
P_{j}=\frac{1}{2}\left(\left.F_{j}\right|_{\nu=c_{1}}+\left.F_{j}\right|_{\nu=-c_{1}}\right), \\
Q_{j}=\frac{1}{2} c_{1}{ }^{-1}\left(\left.F_{j}\right|_{\nu=c_{1}}-\left.F_{j}\right|_{\nu=-c_{1}}\right), \\
P_{j}^{\prime}=\frac{1}{2}\left(\left.F^{\prime}{ }_{j}\right|_{\nu=c_{1}}+\left.F^{\prime}\right|_{\nu=-c_{1}}\right),
\end{gathered}
$$

and

$$
Q^{\prime}{ }_{j}=\frac{1}{2} c_{1}{ }^{-1}\left(\left.F^{\prime}{ }_{j}\right|_{\nu=c_{1}}-\left.F^{\prime}{ }_{j}\right|_{\nu=-c_{1}}\right)
$$


Note that the extrema

$$
\varphi_{ \pm}=2 \pi-\arccos \left[\left(1 \mp \Delta^{\frac{1}{2}}\right) /\left(\hat{r} \hat{r}_{\mathrm{P}}\right)\right]
$$

of the function $g(\varphi)$ transform into $\nu=\mp c_{1}$, respectively.

The derivatives $d \varphi /\left.d \nu\right|_{\nu= \pm c_{1}}$ that appear in the definitions of the coefficients $\left(P_{j}, Q_{j}, P_{j}^{\prime}, Q_{j}^{\prime}\right)$ are indeterminate. Their values must be found by repeated differentiation of Eqs. (15) and (40) with respect to $\nu$ :

$$
\begin{gathered}
(d g / d \varphi)(d \varphi / d \nu)=\nu^{2}-c_{1}^{2} \\
\left(d^{2} g / d \varphi^{2}\right)(d \varphi / d \nu)^{2}+(d g / d \varphi)\left(d^{2} \varphi / d \nu^{2}\right)=2 \nu,
\end{gathered}
$$

etc., and the evaluation of the resulting relations at $\nu= \pm c_{1}$. This procedure, which amounts to applying l'Hôpital's rule, yields

$$
d \varphi /\left.d \nu\right|_{\nu= \pm c_{1}}=\left(2 c_{1} \hat{R}_{\mp}\right)^{\frac{1}{2}} / \Delta^{\frac{1}{4}} .
$$

Using $\partial^{2} g /\left.\partial \varphi^{2}\right|_{\varphi_{ \pm}}=\mp \Delta^{1 / 2} / \hat{R}_{ \pm}$and Eq. (46)), we find from Eq. (42b) that

$$
\left.F_{j}\right|_{\nu= \pm c_{1}}= \pm\left. 2 c_{1} f_{j}\right|_{\nu= \pm c_{1}},
$$

in which $f_{j}=(\mathrm{d} \varphi / \mathrm{d} \nu) h_{j}$ are the functions earlier encountered in the evaluation of $G_{j}$ in Refs. [8] and [9]. Hence, $P_{j}=2 c_{1}{ }^{2} q_{j}$ and $Q_{j}=2 p_{j}$, where $p_{j}$ and $q_{j}$ are precisely the same as the coefficients in Eqs. (20) that are approximated in Eqs. (23)-(26) (see Ref. [9]).

We now need to evaluate $\partial G_{j} / \partial \hat{\varphi}$ only outside the bifurcation surface, i.e., for $|\chi|>1$ [see Eqs. (21) and (36)]. In this region, the argument of the $\delta$ function in Eq. (43a) has a single zero at

$$
\nu=\nu^{*}=2 c_{1} \operatorname{sgn}(\chi) \cosh \left(\frac{1}{3} \operatorname{arccosh}|\chi|\right), \quad|\chi| \geq 1,
$$

(see Appendix A of Ref. [8]). Outside the bifurcation surface, therefore, Eq. (43a) yields

$$
\left.\left(\frac{\partial G_{j}}{\partial \hat{\varphi}}\right)^{\text {out }} \simeq \frac{1}{\left|\nu^{2}-c_{1}{ }^{2}\right|}\left[-\frac{P_{j}+Q_{j} \nu}{\left(\nu^{2}-c_{1}{ }^{2}\right)^{2}}+\frac{P^{\prime}{ }_{j}+Q^{\prime}{ }_{j} \nu}{\nu^{2}-c_{1}{ }^{2}}\right]\right|_{\nu^{*}} .
$$

Keeping only the first term in this expresssion, which is dominant when $c_{1} \ll 1$, we obtain

$$
\left(\frac{\partial G_{j}}{\partial \hat{\varphi}}\right)^{\text {out }} \simeq-\frac{2 \sinh ^{3}\left(\frac{1}{3} \operatorname{arccosh}|\chi|\right)}{c_{1}^{5}\left(\chi^{2}-1\right)^{3 / 2}}\left[c_{1} q_{j}+2 p_{j} \operatorname{sgn}(\chi) \cosh \left(\frac{1}{3} \operatorname{arccosh}|\chi|\right)\right],
$$

in which $p_{j}(r, z)$ and $q_{j}(r, z)$ assume the values given in Eqs. (23) $-(26)$ when $\hat{R}_{\mathrm{P}} \gg 1$. 
Evaluation of the right-hand side of Eq. (50) at $\phi=\phi_{ \pm}$now yields the following term that appears in the expression for $L_{j}{ }^{\text {edge }}$ :

$$
\begin{aligned}
\left.\exp (-\mathrm{i} \mu \hat{\varphi})\left(\partial G_{j} / \partial \hat{\varphi}\right)^{\text {out }}\right|_{\phi_{-}} ^{\phi_{+}} \simeq & -\left(\frac{2}{3}\right)^{3} c_{1}^{-5} \exp \left[-\mathrm{i} \mu\left(\hat{\varphi}_{\mathrm{P}}+c_{2}\right)\right] \\
& \times\left[p_{j} \cos \left(\frac{2}{3} \mu c_{1}^{3}\right)-\frac{1}{2} \mathrm{i} c_{1} q_{j} \sin \left(\frac{2}{3} \mu c_{1}^{3}\right)\right]
\end{aligned}
$$

The asymptotic expansions of $G_{j}{ }^{\text {out }}$ and $\left(\partial G_{j} / \partial \hat{\varphi}\right)^{\text {out }}$ given in Eqs. (20c) and (50) are for small $c_{1}$. To be consistent, we must likewise replace the expression that is found by inserting $G_{j}{ }^{\text {out }}$ and $\left(\partial G_{j} / \partial \hat{\varphi}\right)^{\text {out }}$ in Eq. (36) with the leading term in its expansion in powers of $c_{1}$. The result is

$$
L_{j}^{\text {edge }} \simeq-2^{1 / 3}\left(\frac{2}{3}\right)^{3} R_{\mathrm{P}}{ }^{-1} \bar{p}_{j} c_{1}^{-5} \exp \left(\mathrm{i} \Omega \varphi_{c} / \omega\right) \sum_{\mu=\mu_{ \pm}} \mu \exp \left[-\mathrm{i} \mu\left(\hat{\varphi}_{\mathrm{P}}+\phi_{-}\right)\right]
$$

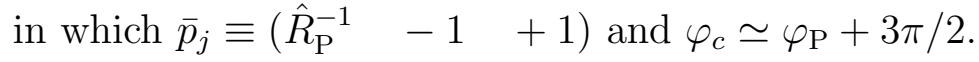

The far-field value of $c_{1}$ close to the cusp curve of the bifurcation surface (where $\Delta=0$ ) is given by

$$
c_{1} \simeq 2^{-\frac{1}{3}} \hat{R}_{\mathrm{P}}^{-1} \Delta^{\frac{1}{2}}
$$

[see Eq. (22)]. Inserting Eq. (52) in Eq. (35) and using Eq. (153), we finally arrive at

$$
\begin{aligned}
\left(\partial \mathbf{B} / \partial \hat{\varphi}_{\mathrm{P}}\right)^{\mathrm{ns}} \simeq & 2\left(\frac{2}{3}\right)^{3} \hat{R}_{\mathrm{P}}^{4} \exp \left[\mathrm{i}\left(\Omega \varphi_{c} / \omega-\pi / 2\right)\right] \sum_{\mu=\mu_{ \pm}} \mu \exp \left(-\mathrm{i} \mu \hat{\varphi}_{\mathrm{P}}\right) \\
& \times \sum_{j=1}^{3} \bar{p}_{j} \int_{\Delta \geq 0} \hat{r} \mathrm{~d} \hat{r} \mathrm{~d} \hat{z} \Delta^{-\frac{5}{2}} \mathbf{u}_{j} \exp \left(-\mathrm{i} \mu \phi_{-}\right) .
\end{aligned}
$$

As in Ref. [10], the integral over $\hat{r}$ in this expression may be evaluated by contour integration. Since the singularity of its integrand at $\Delta=0$ is not integrable, however, the contour integral that passes through this singularity needs to be in addition regularized.

\section{Regularization of the integral over the radial extent of the source}

The kernel of the integral in Eq. (54) has the same phase but a different amplitude compared to that of the integral encountered in Eq. (19) of Ref. [10]. Hence, the asymptotic evaluation of integral (54) entails the same techniques as those used before, but the regularization of this integral requires an extension of the procedure followed in Ref. [10].

The function $\phi_{-}(\hat{r}, \hat{z})$ in the phase of the integrand in Eq. (54) is stationary as a function of $\hat{r}$ at

$$
\hat{r}=\hat{r}_{C}(\hat{z}) \equiv\left\{\frac{1}{2}\left(\hat{r}_{\mathrm{P}}^{2}+1\right)-\left[\frac{1}{4}\left(\hat{r}_{\mathrm{P}}^{2}-1\right)^{2}-\left(\hat{z}-\hat{z}_{\mathrm{P}}\right)^{2}\right]^{1 / 2}\right\}^{1 / 2}
$$


When the observer is located in the far zone, this one-dimensional locus of stationary points coincides with the locus,

$$
\hat{r}=\hat{r}_{S} \equiv\left[1+\left(\hat{z}-\hat{z}_{\mathrm{P}}\right)^{2} /\left(\hat{r}_{\mathrm{P}}^{2}-1\right)\right]^{1 / 2}
$$

of source points that approach the observer with the speed of light and zero acceleration at the retarded time, i.e., with the projection $\Delta=0$ of the cusp curve of the bifurcation surface onto the $(r, z)$ plane (see Fig. 4 of Ref. [10]). For $\hat{R}_{\mathrm{P}} \gg 1$, the separation $\hat{r}_{C}-\hat{r}_{S}$ vanishes as $\hat{R}_{\mathrm{P}}^{-2}$ [see Eq. (69) below] and both $\hat{r}_{C}$ and $\hat{r}_{S}$ assume the value $\csc \theta_{\mathrm{P}}$.

It follows from Eq. (18) that at the stationary point $\hat{r}=\hat{r}_{C}$,

$$
\left.\phi_{-}\right|_{\hat{r}=\hat{r}_{C}} \equiv \phi_{C}=\hat{R}_{C}+\varphi_{C}-\varphi_{\mathrm{P}}
$$

$\partial \phi_{-} /\left.\partial \hat{r}\right|_{\hat{r}=\hat{r}_{C}}=0$, and

$$
\partial^{2} \phi_{-} /\left.\partial \hat{r}^{2}\right|_{\hat{r}=\hat{r}_{C}} \equiv a=-\hat{R}_{C}^{-1}\left[\left(\hat{r}_{\mathrm{P}}^{2}-1\right)\left(\hat{r}_{C}^{2}-1\right)^{-1}-2\right],
$$

where

$$
\varphi_{C}=\varphi_{\mathrm{P}}+2 \pi-\arccos \left(\hat{r}_{C} / \hat{r}_{\mathrm{P}}\right) \quad \text { and } \quad \hat{R}_{C}=\hat{r}_{C}\left(\hat{r}_{\mathrm{P}}^{2}-\hat{r}_{C}^{2}\right)^{\frac{1}{2}}
$$

For observation points of interest to us (the observation points located outside the plane of rotation, $\theta_{\mathrm{P}} \neq \pi / 2$, in the far zone, $\hat{R}_{\mathrm{P}} \gg 1$ ), the parameter $a$ in Eq. (158) has a value whose magnitude increases with increasing $\hat{R}_{\mathrm{P}}$ :

$$
a \simeq-\hat{R}_{\mathrm{P}} \sin ^{4} \theta_{\mathrm{P}} \sec ^{2} \theta_{\mathrm{P}}
$$

In other words, the phase function $\phi_{-}$is more sharply peaked at its maximum, the farther the observation point is from the source. This property of the phase function $\phi_{-}$distinguishes the asymptotic analysis that will be presented in this section from those commonly encountered in radiation theory. What turns out to play the role of a large parameter in this asymptotic expansion is distance $\left(\hat{R}_{\mathrm{P}}\right)$, not frequency $\left(\mu_{ \pm} \omega\right)$.

The first step in the asymptotic analysis of the integral in Eq. (154) is to introduce a change of variable $\xi=\xi(\hat{r}, \hat{z})$ that replaces the original phase $\phi_{-}$of the integrand by as simple a polynomial as possible [22]. This transformation should be one-to-one and should preserve the number and nature of the stationary points of the phase. Since $\phi_{-}$has a single isolated stationary point at $\hat{r}=\hat{r}_{C}(\hat{z})$, it can be cast into a canonical form by means of the following transformation:

$$
\phi_{-}(\hat{r}, \hat{z})=\phi_{C}(\hat{z})+\frac{1}{2} a(\hat{z}) \xi^{2}
$$


in which $a$ is the coefficient given in Eqs. (58) and (60).

The integral in Eq. (54) can thus be written as

$$
\int_{\Delta \geq 0} \hat{r} \mathrm{~d} \hat{r} \mathrm{~d} \hat{z} \Delta^{-5 / 2} \mathbf{u}_{j} \exp \left(-\mathrm{i} \mu \phi_{-}\right)=\int_{\xi \geq \xi_{S}} \mathrm{~d} \hat{z} \mathrm{~d} \xi F(\xi, \hat{z}) \exp \left(\mathrm{i} \alpha \xi^{2}\right),
$$

in which

$$
F(\xi, \hat{z}) \equiv \hat{r} \Delta^{-5 / 2} \mathbf{u}_{j}(\partial \hat{r} / \partial \xi) \exp \left(-\mathrm{i} \mu \phi_{C}\right)
$$

with

$$
\partial \hat{r} / \partial \xi=a \xi \hat{r} \hat{R}_{-}\left(\hat{r}^{2}-1-\Delta^{1 / 2}\right)^{-1}
$$

and $\alpha \equiv-\mu a / 2$. The stationary point $\hat{r}=\hat{r}_{C}$ and the boundary point $\hat{r}=\hat{r}_{S}$ respectively map onto $\xi=0$ and

$$
\xi=\xi_{S} \equiv-\left[2 a^{-1}\left(\phi_{S}-\phi_{C}\right)\right]^{1 / 2},
$$

where

$$
\left.\phi_{S} \equiv \phi_{-}\right|_{\hat{r}=\hat{r}_{S}}=2 \pi-\arccos \left[1 /\left(\hat{r}_{S} \hat{r}_{\mathrm{P}}\right)\right]+\left(\hat{r}_{S}^{2} \hat{r}_{\mathrm{P}}^{2}-1\right)^{1 / 2}
$$

The upper limit of integration in Eq. (62) is determined by the image of the support of the source density ( $\mathbf{s}$ in $\mathbf{u}_{j}$ ) under the transformation (61).

By substituting the value of $r_{\mathrm{C}}$ from Eq. (55) in Eq. (17), we find that $\Delta^{1 / 2}=\hat{r}^{2}-1$ at C. Thus, the Jacobian $\partial \hat{r} / \partial \xi$ of the above transformation is indeterminate at $\xi=0$. Its value at this critical point must be found by repeated differentiation of Eq. (61) with respect to $\xi$,

$$
\begin{gathered}
\left(\partial \phi_{-} / \partial \hat{r}\right)(\partial \hat{r} / \partial \xi)=a \xi \\
\left(\partial^{2} \phi_{-} / \partial \hat{r}^{2}\right)(\partial \hat{r} / \partial \xi)^{2}+\left(\partial \phi_{-} / \partial \hat{r}\right)\left(\partial^{2} \hat{r} / \partial \xi^{2}\right)=a,
\end{gathered}
$$

and the evaluation of the resulting relation (68) at $\hat{r}=\hat{r}_{C}$ with the aid of Eq. (58). This procedure, which amounts to applying l'Hôpital's rule, yields $\partial \hat{r} /\left.\partial \xi\right|_{\xi=0}=1$ : a result we could have anticipated from the coincidence of transformation (61) with the Taylor expansion of $\phi_{-}$about $\hat{r}=\hat{r}_{C}$ to within the leading order. Correspondingly, the amplitude $F(\xi)$ in Eq. (63) has the value $\left.\hat{r}_{\mathrm{C}}\left(\hat{r}_{\mathrm{C}}^{2}-1\right)^{-5} \mathbf{u}_{j}\right|_{\hat{r}=\hat{r}_{\mathrm{C}}} \exp \left(-\mathrm{i} \mu \phi_{\mathrm{C}}\right)$ at the critical point $\mathrm{C}$.

To an observer in the far field $\left(\hat{R}_{\mathrm{P}} \gg 1\right)$, the phase of the integrand on the right-hand side of Eq. (62) is rapidly oscillating, irrespective of how low the harmonic numbers $\mu_{ \pm}$(i.e., the radiation frequencies $\left.\mu_{ \pm} \omega\right)$ may be. The leading contribution to the asymptotic value 
of integral (62) from the stationary point $\xi=0$ can therefore be determined by the method of stationary phase. However, in the limit $\hat{R}_{\mathrm{P}} \rightarrow \infty, \xi_{S}$ reduces to

$$
\xi_{S} \simeq-3^{-1 / 2} \cos ^{4} \theta_{\mathrm{P}} \csc ^{5} \theta_{\mathrm{P}} \hat{R}_{\mathrm{P}}^{-2}
$$

so that the stationary point $\xi=0$ is separated from the boundary point $\xi=\xi_{S}$ by an interval of the order of $\hat{R}_{\mathrm{P}}^{-2}$ only. We therefore need to employ a technique for the asymptotic analysis of integral (62) that is capable of handling the contributions from both $\hat{r}_{\mathrm{C}}$ and $\hat{r}_{\mathrm{S}}$ simultaneously.

\section{E. Contours of steepest descent}

The technique we shall employ for this purpose is the method of steepest descents [22]. We regard the variable of integration in

$$
J(\hat{z}) \equiv \int_{\xi_{S}}^{\xi>} \mathrm{d} \xi F(\xi, \hat{z}) \exp \left(\mathrm{i} \alpha \xi^{2}\right)
$$

as complex, i.e., write $\xi=u+\mathrm{i} v$, and invoke Cauchy's integral theorem to deform the original path of integration into the contours of steepest descent that pass through each of the critical points $\xi=\xi_{S}, \xi=0$ and $\xi=\xi_{>}$. Here, we have introduced the real variable $\xi_{>}(\hat{z})$ to designate the image of $\hat{r}_{>}$under transformation (61), i.e., the boundary of the support of the source term $\mathbf{u}_{j}$ that appears in the amplitude $F(\xi, \hat{z})$. We shall only treat the case in which $\mu$ (and hence $\alpha$ ) is positive; $J(\hat{z})$ for negative $\mu$ can then be obtained by taking the complex conjugate of the derived expression and replacing $\phi_{\mathrm{C}}$ with $-\phi_{\mathrm{C}}$ [see Eq. (63) $]$.

The path of steepest descent through the stationary point $\mathrm{C}$ at which $\xi=0$ is given, according to

$$
\mathrm{i} \xi^{2}=-2 u v+\mathrm{i}\left(u^{2}-v^{2}\right)
$$

by $u=v$ when $\alpha$ is positive. If we designate this path by $C_{1}$ (see Fig. 1), then

$$
\begin{gathered}
\int_{C_{1}} \mathrm{~d} \xi F(\xi, \hat{z}) \exp \left(\mathrm{i} \alpha \xi^{2}\right)=\left.(1+\mathrm{i}) \int_{-\infty}^{\infty} \mathrm{d} v F\right|_{\xi=(1+\mathrm{i}) v} \exp \left(-2 \alpha v^{2}\right) \\
\left.\simeq(2 \pi / \mu)^{1 / 2} \exp \left[-\mathrm{i}\left(\mu \phi_{\mathrm{C}}-\pi / 4\right)\right] \mathbf{u}_{j}\right|_{\mathrm{C}} \sin ^{7} \theta_{\mathrm{P}}\left|\sec \theta_{\mathrm{P}}\right|^{9} \hat{R}_{\mathrm{P}}^{-1 / 2}
\end{gathered}
$$

for $\hat{R}_{\mathrm{P}} \gg 1$. Here, we have obtained the leading term in the asymptotic expansion of the above integral for large $\hat{R}_{\mathrm{P}}$ by approximating $\left.F\right|_{\xi=(1+\mathrm{i}) v}$ by its value at $C$, where $v=0$, and 
using Eqs. (55) and (60) to replace $\hat{r}_{\mathrm{C}}$ and $\alpha$ by their values in the far zone. Note that the next term in this asymptotic expansion is smaller by a factor of order $\hat{R}_{\mathrm{P}}^{-1 / 2}$ than this leading term.

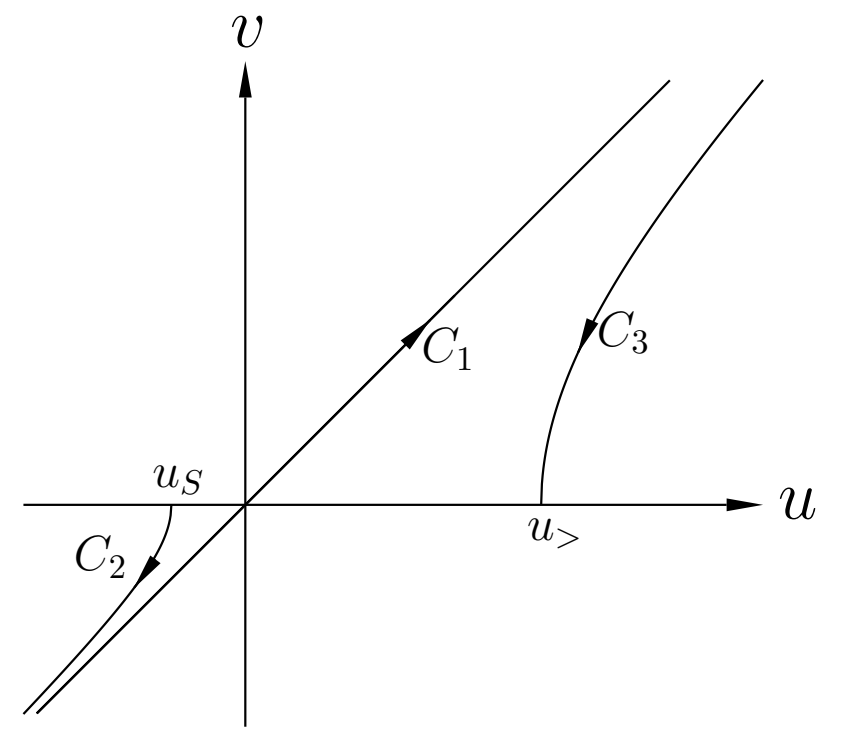

FIG. 1: The integration contours in the complex plane $\xi=u+\mathrm{i} v$. The critical point $\mathrm{C}$ lies at the origin and $u_{S}$ and $u_{>}$are the images under transformation (61) of the radial boundaries $\hat{r}=\hat{r}_{S}(\hat{z})$ and $\hat{r}=\hat{r}_{>}(\hat{z})$ of the part of the source that lies within $\Delta>0$. The contours $C_{1}, C_{2}$, and $C_{3}$ are the paths of steepest descent through the stationary point $\mathrm{C}$ and through the lower and upper boundaries of the integration domain, respectively.

The path of steepest descent through the boundary point $\mathrm{S}$, at which $u \equiv u_{S}=\xi_{S}$ and $v=0$ [see Eqs. (65) and (69)], is given by $u=-\left(v^{2}+u_{S}^{2}\right)^{1 / 2}$, i.e., by the contour designated as $C_{2}$ in Fig. 1, The real part of

$$
\left.\mathrm{i} \xi^{2}\right|_{C_{2}}=2 v\left(v^{2}+u_{S}^{2}\right)^{1 / 2}+\mathrm{i} u_{S}^{2}
$$

is a monotonic function of $v$ and so can be used as a curve parameter for contour $C_{2}$ in place of $v$. If we let $2 v\left(v^{2}+u_{S}{ }^{2}\right)^{1 / 2} \equiv-\tau^{2}$, then it follows from

$$
\left.\xi\right|_{C_{2}}=-\left(u_{S}^{2}+\mathrm{i} \tau^{2}\right)^{1 / 2}
$$

that

$$
\begin{aligned}
\int_{C_{2}} \mathrm{~d} \xi F(\xi, \hat{z}) \exp \left(\mathrm{i} \alpha \xi^{2}\right)=\exp \left[\mathrm{i}\left(\alpha u_{S}^{2}-\pi / 2\right)\right] \\
\quad \times\left.\int_{0}^{\infty} \mathrm{d} \tau \tau\left(u_{S}^{2}+\mathrm{i} \tau^{2}\right)^{-1 / 2} F\right|_{\xi=-\left(u_{S}{ }^{2}+\mathrm{i} \tau^{2}\right)^{1 / 2}} \exp \left(-\alpha \tau^{2}\right)
\end{aligned}
$$


The function $\left.F\right|_{C_{2}}$ in this expression has to be determined by inverting the following version of the original transformation (61):

$$
\phi_{-}(\hat{r}, \hat{z})-\phi_{S}(\hat{z})=\frac{1}{2} \mathrm{i} a \tau^{2}
$$

Here, we have used Eqs. (65) and (174) to rewrite Eq. (61) in terms of $\tau$.

Since the dominant contribution toward the asymptotic value of the above integral for $\hat{R}_{\mathrm{P}} \gg 1$ comes from the vicinity of the boundary point $\mathrm{S}$, where $\tau=0$, the required inversion of transformation (76) can be carried out by means of a Taylor expansion of the phase function $\phi_{-}$in powers of $\tau$ (see Appendix $\mathrm{A}$ ). We find in Appendix $\mathrm{A}$ that the resulting expression for $\left.F(\tau)\right|_{C_{2}}$ diverges at $\tau=0$ as $\tau^{-4}$. Therefore, as in the case of the integral over $\hat{\varphi}$ in Eq. (11), we must regard the divergent integral in Eq. (75) as a generalized function that equals its Hadamard's finite part (see, e.g., Ref. [17]). The procedure for finding the Hadamard finite part of this integral, though lengthy, is straightforward and results in

$$
\begin{aligned}
\mathcal{F}\left\{\int_{C_{2}} \mathrm{~d} \xi F(\xi, \hat{z}) \exp \left(\mathrm{i} \alpha \xi^{2}\right)\right\}= & \left(35 / 6^{4}\right)(2 \pi / \mu)^{1 / 2} \exp \left[-\mathrm{i}\left(\mu \phi_{S}-3 \pi / 4\right)\right] \\
& \times\left.\mathbf{u}_{j}\right|_{S} \sin ^{7} \theta_{\mathrm{P}}\left|\sec \theta_{\mathrm{P}}\right|^{9} \hat{R}_{\mathrm{P}}^{-1 / 2}
\end{aligned}
$$

in the limit $\hat{R}_{\mathrm{P}} \gg 1$ (see Appendix $\underline{\mathrm{A}}$ ).

The path of steepest descent through the boundary point $\xi=\xi_{>}$, at which $u=u_{>}, v=0$, is given by $u=\left(v^{2}+u_{>}\right)^{1 / 2}$, i.e., by the contour designated as $C_{3}$ in Fig. 1. The real part of the exponent

$$
\left.\mathrm{i} \xi^{2}\right|_{C_{3}}=-2 v\left(v^{2}+u_{>}^{2}\right)^{1 / 2}+\mathrm{i} u_{>}^{2}
$$

is again a monotonic function of $v$ and so can be used to parametrize contour $C_{3}$ in place of $v$. If we let $2 v\left(v^{2}+u_{>}^{2}\right)^{1 / 2} \equiv \chi$, then it follows from

$$
\left.\xi\right|_{C_{3}}=\left(u_{>}^{2}+\mathrm{i} \chi\right)^{1 / 2}
$$

that

$$
\begin{aligned}
\int_{C_{3}} \mathrm{~d} \xi F(\xi, \hat{z}) \exp \left(\mathrm{i} \alpha \xi^{2}\right)=\frac{1}{2} \exp \left[\mathrm{i}\left(\alpha u_{>}^{2}-\pi / 2\right)\right] \\
\quad \times\left.\int_{0}^{\infty} \mathrm{d} \chi\left(u_{>}^{2}+\mathrm{i} \chi\right)^{-1 / 2} F\right|_{\xi=\left(u_{>}{ }^{2}+\mathrm{i} \chi\right)^{1 / 2}} \exp (-\alpha \chi) .
\end{aligned}
$$

The asymptotic value of this integral for $\hat{R}_{\mathrm{P}} \gg 1$ receives its dominant contribution from $\chi=0$. Because the function $\left.F\right|_{C_{3}}$ is regular, on the other hand, its value at $\chi=0$ can be 
found by simply evaluating the expression in Eq. (63) at $\hat{r}=\hat{r}_{>}$. The result, for $\hat{R}_{\mathrm{P}} \rightarrow \infty$, is

$$
\left.\left.F\right|_{C_{3}, \chi=0} \simeq \hat{r}_{>}^{2} \hat{R}_{\mathrm{P}}^{-4} \sin ^{4} \theta_{\mathrm{P}} \sec ^{2} \theta_{\mathrm{P}}\left(\hat{r}_{>}^{2} \sin ^{2} \theta_{\mathrm{P}}-1\right)^{-3} \mathbf{u}_{j}\right|_{\hat{r}=\hat{r}_{>}} \exp \left(-\mathrm{i} \mu \phi_{\mathrm{C}}\right) u_{>}
$$

[see Eqs. (17) and (60)]. This in conjunction with Watson's lemma therefore implies that

$$
\begin{aligned}
\int_{C_{3}} \mathrm{~d} \xi F(\xi, \hat{z}) \exp \left(\mathrm{i} \alpha \xi^{2}\right) \simeq & \left.\hat{r}_{>}^{2}\left(\hat{r}_{>}^{2} \sin ^{2} \theta_{\mathrm{P}}-1\right)^{-3} \mathbf{u}_{j}\right|_{\hat{r}=\hat{r}>} \\
& \times \exp \left[-\mathrm{i}\left(\left.\phi_{-}\right|_{\hat{r}=\hat{r}_{>}}+\pi / 2\right)\right] \mu^{-1} \hat{R}_{\mathrm{P}}^{-5},
\end{aligned}
$$

to within the leading order in $\hat{R}_{\mathrm{P}}^{-1}$.

\section{F. Asymptotic value of the gradient of the field for large distances}

The integral in Eq. (70) equals the sum of the three contour integrals in Eqs. (72), (77), and (82); the contributions of the contours that connect $C_{1}$ and $C_{2}$, and $C_{1}$ and $C_{3}$, at infinity (see Fig. 1) are exponentially small compared to those of $C_{1}, C_{2}$, and $C_{3}$ themselves. On the other hand, the leading term in the asymptotic value of the integral over $C_{3}$ decreases (with increasing $\hat{R}_{\mathrm{P}}$ ) much faster than those in the asymptotic values of the integrals over $C_{1}$ and $C_{2}$ : the integral over $C_{3}$ decays as $\hat{R}_{\mathrm{P}}^{-5}$, while the integrals over $C_{1}$ and $C_{2}$ decay as $\hat{R}_{\mathrm{P}}^{-1 / 2}$. According to Eqs. (54), (62), (72) and (77), the leading term in the asymptotic expansion of the contribution $\left(\partial \mathbf{B} / \partial \hat{\varphi}_{\mathrm{P}}\right)^{\text {ns }}$, for large $\hat{R}_{\mathrm{P}}$, is therefore given by

$$
\begin{aligned}
\left(\partial \mathbf{B} / \partial \hat{\varphi}_{\mathrm{P}}\right)^{\mathrm{ns}} \simeq & \frac{1}{3^{7}}\left(35-6^{4} \mathrm{i}\right) \hat{R}_{\mathrm{P}}^{7 / 2} \sin ^{7} \theta_{\mathrm{P}}\left|\sec \theta_{\mathrm{P}}\right|^{9} \exp \left\{\mathrm{i}\left[(\Omega / \omega) \varphi_{\mathrm{C}}+\pi / 4\right]\right\} \\
& \times \sum_{\mu=\mu_{ \pm}}(2 \pi|\mu|)^{1 / 2} \operatorname{sgn}(\mu) \exp \left(\mathrm{i} \frac{\pi}{4} \operatorname{sgn} \mu\right) \\
& \times\left.\sum_{j=1}^{3} \bar{p}_{j} \int_{-\infty}^{\infty} \mathrm{d} \hat{z} \exp \left[-\mathrm{i} \mu\left(\phi_{\mathrm{C}}+\hat{\varphi}_{\mathrm{P}}\right)\right] \mathbf{u}_{j}\right|_{\mathrm{C}}
\end{aligned}
$$

in which $\mu_{ \pm}$can also be negative (see the first paragraph of Section IIIE).

The remaining $\hat{z}$ integration in the above expression for $\left(\partial \mathbf{B} / \partial \hat{\varphi}_{\mathrm{P}}\right)^{\mathrm{ns}}$ amounts to a Fourier decomposition of the source densities $\left.s_{r, \varphi, z}\right|_{\mathrm{C}}$ with respect to $\hat{z}$. Using Eqs. (57)-(59) to replace $\phi_{\mathrm{C}}$ in Eq. (83) by its far-field value

$$
\phi_{\mathrm{C}} \simeq \hat{R}_{\mathrm{P}}-\hat{z} \cos \theta_{\mathrm{P}}+3 \pi / 2,
$$


and using Eq. (12) to write out $\mathbf{u}_{j}$ in terms of $s_{r, \varphi, z}$, we find that

$$
\begin{aligned}
\left(\partial \mathbf{B} / \partial \hat{\varphi}_{\mathrm{P}}\right)^{\mathrm{ns}} \simeq & \frac{1}{3^{7}}\left(35-6^{4} \mathrm{i}\right) \hat{R}_{\mathrm{P}}^{7 / 2} \sin ^{7} \theta_{\mathrm{P}}\left|\sec \theta_{\mathrm{P}}\right|^{9} \\
& \times \exp \left\{\mathrm{i}\left[\pi / 4+(\Omega / \omega)\left(\varphi_{\mathrm{P}}+3 \pi / 2\right)\right]\right\} \\
& \times \sum_{\mu=\mu_{ \pm}}(2 \pi|\mu|)^{1 / 2} \operatorname{sgn}(\mu) \exp \left\{\mathrm{i}\left[\frac{\pi}{4} \operatorname{sgn}(\mu)-\mu\left(\hat{R}_{\mathrm{P}}+\hat{\varphi}_{\mathrm{P}}+3 \pi / 2\right)\right]\right\} \\
& \times\left[\left(\bar{s}_{\varphi} \cos \theta_{\mathrm{P}}-\bar{s}_{z} \sin \theta_{\mathrm{P}}\right) \hat{\mathbf{e}}_{\|}-\bar{s}_{r} \hat{\mathbf{e}}_{\perp}\right]
\end{aligned}
$$

where $\bar{s}_{r, \varphi, z}$ stand for the following Fourier transforms of $\left.s_{r, \varphi, z}\right|_{\mathrm{C}}$ with respect to $\hat{z}$ :

$$
\left.\bar{s}_{r, \varphi, z} \equiv \int_{-\infty}^{\infty} \mathrm{d} \hat{z} s_{r, \varphi, z}(\hat{r}, \hat{z})\right|_{\hat{r}=\csc \theta_{\mathrm{P}}} \exp \left(\mathrm{i} \mu \hat{z} \cos \theta_{\mathrm{P}}\right)
$$

Being the contribution from the source elements that approach the observer with the speed of light and zero acceleration at the retarded time, this expression is valid only at those polar angles $\theta_{\mathrm{P}}$ within the interval $\arccos \left(1 / \hat{r}_{<}\right) \leq\left|\theta_{\mathrm{P}}-\pi / 2\right| \leq \arccos \left(1 / \hat{r}_{>}\right)$for which $\left.s_{r, \varphi, z}\right|_{\hat{r}=\csc \theta_{\mathrm{P}}}$ is nonzero, i.e., at those observation points (outside the plane of rotation) the cusp curve of whose bifurcation surface intersects the source distribution. At these polar angles, the above expression for $\left(\partial \mathbf{B} / \partial \hat{\varphi}_{\mathrm{P}}\right)^{\mathrm{ns}}$ constitutes the dominant contribution toward the gradient $\partial \mathbf{B} / \partial \hat{\varphi}_{\mathrm{P}}$ of the magnetic field of the radiation (see Subsection ऑIIB).

\section{EVALUATION OF THE BOUNDARY TERM IN THE RETARDED SOLU- TION TO THE WAVE EQUATION GOVERNING THE FIELD}

Let the boundary $\Sigma$ in the second term of Eq. (8) be a large sphere enclosing the source. The element $\mathrm{d} \mathbf{S}$ of area for this boundary then has the form $\rho^{2} \sin \theta \mathrm{d} \theta \mathrm{d} \varphi \hat{\mathbf{e}}_{\rho}$, where $(\rho, \varphi, \theta)$ are the spherical polar coordinates in the space of source points, i.e., are related to the cylindrical polar coordinates $(r, \varphi, z)$ we have been using by

$$
\rho \equiv\left(r^{2}+z^{2}\right)^{1 / 2}, \quad \theta \equiv \arctan (r / z)
$$

and $\hat{\mathbf{e}}_{\rho}$ is a unit vector in the direction of increasing $\rho$. Inserting this in the integrand of the boundary contribution in Eq. (8), we obtain

$$
\mathbf{B}_{\text {boundary }}=\rho^{2} \int \mathrm{d} t \int_{\Sigma} \mathrm{d} \varphi \mathrm{d} \theta \sin \theta\left(G \partial \mathbf{B} /\left.\partial R_{\mathrm{P}}\right|_{R_{\mathrm{P}}=\rho}-\mathbf{B} \partial G / \partial \rho\right),
$$

since $\left(\hat{\mathbf{e}}_{\rho} \cdot \nabla\right) \mathbf{B}=\partial \mathbf{B} / \partial \rho=\partial \mathbf{B} /\left.\partial R_{\mathrm{P}}\right|_{R_{\mathrm{P}}=\rho}$. We will be identifying the magnetic field $\mathbf{B}$ and its gradient on the boundary $\Sigma$ with those of the radiation field that arises from source (9). 
These terms, which act as densities of two-dimensional sources in Eq. (88), both have rigidly rotating distribution patterns, i.e., are functions of $t$ in the combination $\hat{\varphi}=\varphi-\omega t$ only [see Eq. (85)].

Once the free-space Green's function (5) is inserted in Eq. (88), we can therefore cast it in the same form as Eq. (11) by changing the integration variable $t$ to $\hat{\varphi}$, resulting in

$$
\mathbf{B}_{\text {boundary }}=\hat{\rho}^{2} \int_{\Sigma} \mathrm{d} \hat{\varphi} \mathrm{d} \theta \sin \theta\left(G_{b} \partial \mathbf{B} /\left.\partial \hat{R}_{\mathrm{P}}\right|_{R_{\mathrm{P}}=\rho}-\mathbf{B} \partial G_{b} / \partial \hat{\rho}\right)
$$

with

$$
G_{b} \equiv \int \mathrm{d} \varphi \hat{R}^{-1} \delta(g-\phi)
$$

where $\hat{\rho}=\rho \omega / c$, and $g$ and $\phi$ are the same functions as those appearing in Eqs. (13) $-(15)$. Equation (90) implies that

$$
\begin{aligned}
G_{b} & =\sum_{\varphi=\varphi_{j}} \frac{1}{\hat{R}|\partial g / \partial \varphi|} \\
& =\sum_{\varphi=\varphi_{j}}\left|\hat{R}+\hat{\rho} \hat{R}_{\mathrm{P}} \sin \theta \sin \theta_{\mathrm{P}} \sin \left(\varphi_{j}-\varphi_{\mathrm{P}}\right)\right|^{-1},
\end{aligned}
$$

where $\varphi_{j}$ are the solutions of the transcendental equation $g(\varphi)=\phi$ [see Eq. (15)]. For $\hat{\rho} \gg 1$, the number of retarded positions $\varphi_{j}$ of the rapidly rotating distribution patterns of $\left.\mathbf{B}\right|_{\Sigma}$ and $\partial \mathbf{B} /\left.\partial \hat{R}_{\mathrm{P}}\right|_{\Sigma}$ that contribute toward the value of $G_{b}$ can be appreciably larger than three (see Ref. [7]).

The expression in Eq. (11) for the magnetic field $\mathbf{B}$ depends on $R_{\mathrm{P}}$ through $\partial G_{j} / \partial \hat{\varphi}$ only, so that

$$
\begin{aligned}
\partial \mathbf{B} / \partial \hat{R}_{\mathrm{P}}= & -\frac{1}{2} \mathrm{i}(\omega / c)^{2} \sum_{\mu=\mu_{ \pm}} \int_{V} r \mathrm{~d} r \mathrm{~d} \hat{\varphi} \mathrm{d} z \mu \exp (-\mathrm{i} \mu \hat{\varphi}) \\
& \times \sum_{j=1}^{3} \mathbf{u}_{j} \partial^{2} G_{j} / \partial \hat{R}_{\mathrm{P}} \partial \hat{\varphi} .
\end{aligned}
$$

with

$$
\partial^{2} G_{j} / \partial \hat{R}_{\mathrm{P}} \partial \hat{\varphi}=-(\omega / c) \int_{\Delta \varphi} \mathrm{d} \varphi h_{j}(\varphi) \hat{R}^{-1}\left[\delta^{\prime \prime}(g-\phi)-\hat{R}^{-1} \delta^{\prime}(g-\phi)\right] \partial \hat{R} / \partial \hat{R}_{\mathrm{P}}
$$

where $h_{j}$ are the functions defined in Eq. (38), and a prime denotes differentiation of the delta function with respect to its argument [see Eq. (37)].

It follows from a comparison of the calculations described in Subsections IIIA and IIC that the contribution of the second term on the right-hand side of Eq. (93) toward the value 
of $\partial \mathbf{B} / \partial \hat{R}_{\mathrm{P}}$ is by a factor of the order of $\hat{R}_{\mathrm{P}}^{-4}$ smaller than that of the first term. Ignoring this small term, we obtain an expression for $-\partial^{2} G_{j} / \partial \hat{R}_{\mathrm{P}} \partial \hat{\varphi}$ that differs from the expression for $\partial^{2} G_{j} / \partial \hat{\varphi}^{2}$ only by the factor

$$
\partial \hat{R} / \partial \hat{R}_{\mathrm{P}}=\hat{R}^{-1}\left[\hat{R}_{\mathrm{P}}-\hat{z} \cos \theta_{\mathrm{P}}-\hat{r} \sin \theta_{\mathrm{P}} \cos \left(\varphi-\varphi_{\mathrm{P}}\right)\right]
$$

[see Eq. (14)], which reduces to 1 in the limit $\hat{R}_{\mathrm{P}} \gg 1$. Correspondingly, the leading contribution, $\left(\partial \mathbf{B} / \partial \hat{R}_{\mathrm{P}}\right)^{\mathrm{ns}}$, toward the value of $\partial \mathbf{B} / \partial \hat{R}_{\mathrm{P}}$ is given by an expression identical to that in Eq. (85) for $\left(\partial \mathbf{B} / \partial \varphi_{\mathrm{P}}\right)^{\mathrm{ns}}$, except that it is multiplied by -1 (see Section $\underline{\text { III }}$ ). The absolute value of $\partial \mathbf{B} / \partial \hat{R}_{\mathrm{P}}$ is therefore of the same order of magnitude as that of $\partial^{2} G_{j} / \partial \hat{R}_{\mathrm{P}} \partial \hat{\varphi}$, and so increases as $\hat{R}_{\mathrm{P}}^{7 / 2}$ (see Subsection $\llbracket$ IIF).

We already know that the radiation subbeams that are generated by the superluminal source (9) have the widths $\delta \theta \sim \hat{R}_{\mathrm{P}}^{-1}$ and $\delta \hat{\varphi} \sim \hat{R}_{\mathrm{P}}^{-3}$ (see Ref. [10]). In the limit where the values of $\hat{\rho}$ and $\hat{R}_{\mathrm{P}}$ (i.e., the positions of the boundary and the observer) tend to infinity independently of each other, the Green's function $G_{b}$ reduces to

$$
G_{b} \simeq \sum_{\varphi=\varphi_{j}}\left[\hat{\rho} \hat{R}_{\mathrm{P}} \sin \theta \sin \theta_{\mathrm{P}}\left|\sin \left(\varphi_{j}-\varphi_{\mathrm{P}}\right)\right|\right]^{-1}
$$

Hence, the absolute values of $G_{b}$ and $\partial G_{b} / \partial \hat{\rho}$ diminish with distance as $\left(\hat{\rho} \hat{R}_{\mathrm{P}}\right)^{-1}$ and $\hat{\rho}^{-2} \hat{R}_{\mathrm{P}}^{-1}$, respectively. Since $\left|\partial \mathbf{B} / \partial \hat{R}_{\mathrm{P}}\right|$ increases as $\hat{R}_{\mathrm{P}}^{7 / 2}$ while $|\mathbf{B}|$ decreases as $\hat{R}_{\mathrm{P}}^{-1 / 2}$, this means that, of the two terms inside the parantheses in Eq. (89), the second is negligibly smaller than the first. Inserting the orders of magnitude of the remaining factors in Eq. (89), in the order in which they appear in the first term of this equation, we obtain

$$
\begin{aligned}
\left|\mathbf{B}_{\text {boundary }}\right| & \sim \hat{\rho}^{2} \times \hat{\rho}^{-3} \times \hat{\rho}^{-1} \times\left(\hat{\rho} \hat{R}_{\mathrm{P}}\right)^{-1} \times \hat{\rho}^{7 / 2} \\
& \sim \hat{\rho}^{1 / 2} \hat{R}_{\mathrm{P}}^{-1}
\end{aligned}
$$

Thus, the absolute value of the boundary contribution toward the value of the field decays as $\hat{R}_{\mathrm{P}}^{-1 / 2}$ when the radius $\rho$ of the spherical boundary $\Sigma$ and the coordinate $R_{\mathrm{P}}$ of the observation point $P$ are both large and of the same order of magnitude.

\section{CONCLUDING REMARKS}

The unaviodably lengthy calculation that we have presented in Sections [II and IV] both lends support to the conclusions of Ref. [10], on the morphology of the radiation beam that 
is generated by a polarization current with a superluminally rotating distribution pattern, and clarifies a fundamental issue concerning the method of calculating the radiation field of such a polarization current, which has been the source of a long-standing controversy 13 17]. This calculation establishes

(i) that the absolute values of both the radial component $\partial \mathbf{B} / \partial \hat{R}_{\mathrm{P}}$ and the azimuthal or temporal component $\partial \mathbf{B} / \partial \hat{\varphi}_{\mathrm{P}}$ of the gradient of the radiation field that is generated by the superluminal source distribution (9) in the far zone are of the order of $\hat{R}_{\mathrm{P}}^{7 / 2}$ at any observation point within the overall radiation beam arising from this source [23],

(ii) that the angular distribution of the emitted field contains sharply focused structures, i.e., that the overall radiation beam is composed of an incoherent superposition of rapidly narrowing subbeams [11],

(iii) that the boundary contribution toward the the solution of the wave equation governing the field decays as $\hat{R}_{\mathrm{P}}^{-1 / 2}$ as the boundary tends to infinity, i.e., that the second term in Eq. (8) is by a factor of the order of $\hat{R}_{\mathrm{P}}^{1 / 2}$ greater than the first term in this equation for a $\Sigma$ that lies in $\hat{R}_{\mathrm{P}} \gg 1$,

(iv) that the discrepancy between the predictions of Eq. (2) and Eq. (17) disappears once one includes the boundary term that is normally neglected in solution (8), and

(v) that Hannay's erroneous contention that the field of a rotating superluminal source should diminish as $R_{\mathrm{P}}{ }^{-1}$, as does a conventional radiation field [13 16], stems from his having neglected the boundary term in the solution [Eq. (8)] to the wave equation governing the field [Eq. (17)].

The sharply focused radiation pulses encountered in the present analysis are in fact observed in astronomical objects that are thought to contain superluminal sources. The radio emission received from pulsars is composed (often entirely [24]) of a collection of socalled giant pulses whose widths are as narrow as $1 \mathrm{~ns}$ [25] and whose brightness temperatures are as high as $10^{39} \mathrm{~K}$ [26]. Hankins et al [25] note the puzzling brightness of these pulses:

The plasma structures responsible for these emissions must be smaller than one meter in size, making them by far the smallest objects ever detected and resolved outside the Solar System, and the brightest transient radio sources in the sky. 
The small size of the emitting structures reflects, in the present context, the narrowing (as $R_{\mathrm{P}}{ }^{-2}$ and $R_{\mathrm{P}}{ }^{-3}$, respectively) of the radial and azimuthal dimensions of the filamentary part of the source that approaches the observer at $\mathrm{P}$ with the speed of light and zero acceleration at the retarded time [10]. This, together with the nonspherical decay of the individual subbeams generated by such filaments (as $R_{\mathrm{P}}{ }^{-1 / 2}$ instead of $R_{\mathrm{P}}{ }^{-1}$ ), easily accounts for the observationally inferred values of the brightness temperature of the giant pulses.

The azimuthal (or temporal) gradient of the intensity of these pulses often appears infinitely sharp at either their leading or trailing edges (see Fig. 1 of Ref. [25]). Correspondingly, the emission mechanism discussed in this paper sets no upper limit on the gradient $\partial / \partial \hat{\varphi}_{\mathrm{P}}$ of the radiation field (i.e., on the sharpness of the leading or trailing edge of the pulse), if the length scale of spatial or temporal variations of its source are comparable with $\hat{R}_{\mathrm{P}}^{-3}$. According to the superluminal model of pulsars [27] (to which the present findings apply), the more distant a pulsar, the narrower and brighter its giant pulses should be.

\section{Acknowledgements}

H. A. thanks Boris Bolotovskii for his help and encouragement, and Alexander Schekochihin and Janusz Gill for their stimulating questions and comments. A. A. is supported by the Royal Society. J. S., J. F., and A. S. are supported by U.S. Department of Energy grant LDRD 20050540ER.

\section{APPENDIX A: HADAMARD'S FINITE PART OF THE DIVERGENT CONTRI- BUTION FROM THE INTEGRAL OVER THE CONTOUR $C_{2}$}

Our first task in this Appendix is to make the dependence of the integrand of integral (75) on the integration variable $\tau$ explicit. This entails (i) inverting Eq. (76) in the vicinity of the critical point $\tau=0$ to obtain $\hat{r}$ as a function of $\tau$ for a fixed value of $\hat{z}$, and (ii) expanding the function $\left.F\right|_{C_{2}}$ that appears in the integrand of integral (75) in powers of $\tau$. Next, we calculate the Hadamard finite part of the resulting integral (whose integrand turns out to diverge as $\tau^{-4}$ at $\tau=0$ ) by following the standard procedure used in the literature on generalized functions [19]. 
Because it contains the factor

$$
\Delta^{1 / 2}=\left(\hat{r}^{2}-1\right)^{1 / 2}\left(\hat{r}^{2}-\hat{r}_{S}^{2}\right)^{1 / 2},
$$

the function $\phi_{-}(\hat{r}, \hat{z})$ is not analytic at $\hat{r}=\hat{r}_{S}$ [see Eqs. (17), (18) and (56)]. If, however, we eliminate $\hat{r}$ in $\phi_{-}$in favour of

$$
\eta \equiv\left(\hat{r}^{2}-\hat{r}_{S}^{2}\right)^{1 / 2},
$$

then the resulting function

$$
\begin{aligned}
\phi_{-}(\eta, \hat{z})= & \left\{\hat{r}_{\mathrm{P}}^{2}\left(\hat{r}_{S}^{2}+\eta^{2}\right)-\left[1+\left(\hat{r}_{\mathrm{P}}^{2}-1\right)^{1 / 2} \eta\right]^{2}\right\}^{1 / 2} \\
& +2 \pi-\arccos \left\{\hat{r}_{\mathrm{P}}^{-1}\left(\hat{r}_{S}^{2}+\eta^{2}\right)^{-1 / 2}\left[1+\left(\hat{r}_{\mathrm{P}}^{2}-1\right)^{1 / 2} \eta\right]\right\}
\end{aligned}
$$

can be expanded into a Taylor series about $\eta=0$ to obtain

$$
\begin{aligned}
\phi_{-}= & \phi_{S}+\frac{1}{2} \hat{R}_{\mathrm{P}}^{-1} \cos ^{2} \theta_{\mathrm{P}} \eta^{2}-\frac{1}{3} \sin ^{3} \theta_{\mathrm{P}} \eta^{3}+\frac{1}{8} \hat{R}_{\mathrm{P}}^{-3} \cos ^{2} \theta_{\mathrm{P}}\left(5 \sin ^{2} \theta_{\mathrm{P}}-1\right) \eta^{4} \\
& +\frac{1}{5} \sin ^{5} \theta_{\mathrm{P}} \eta^{5}+\cdots .
\end{aligned}
$$

Here, the coefficients in this series are approximated by their dominant values for $\hat{R}_{\mathrm{P}} \gg 1$, and the coordinate $\hat{r}_{\mathrm{S}}$ that appears in them is replaced by its value $\csc \theta_{\mathrm{P}}$ at the radius from which the main contributions toward the field in the far zone arise [see Eq. (56)]. Equation (A4), in conjunction with Eq. (76), provides us with an analytic expression for $\tau(\eta)$ that we can invert to find $\eta$ (and hence $\hat{r}$ ) as a function of $\tau$.

Repeated differentiations of Eq. (76) with respect to $\tau$ result in

$$
\begin{gathered}
\left(\partial \phi_{-} / \partial \eta\right)(\partial \eta / \partial \tau)=\mathrm{i} a \tau \\
\left.\left(\partial \phi_{-} / \partial \eta\right)\left(\partial^{2} \eta / \partial \tau^{2}\right)+\partial^{2} \phi_{-} / \partial \eta^{2}\right)(\partial \eta / \partial \tau)^{2}=\mathrm{i} a,
\end{gathered}
$$

and so on, which when evaluated at $\mathrm{S}$ (where $\eta=\tau=0$ ), yield $\partial \eta /\left.\partial \tau\right|_{S}, \partial^{2} \eta /\left.\partial \tau^{2}\right|_{S}$, etc., in terms of the known derivatives $\partial \phi_{-} /\left.\partial \eta\right|_{S}, \partial^{2} \phi_{-} /\left.\partial \eta^{2}\right|_{S}$, etc., that constitute the coefficients in Eq. (A4). Using these derivatives of $\eta$ at $\mathrm{S}$, we can therefore write down the Taylor expansion of $\eta$ in powers of $\tau$ :

$$
\hat{\eta}=\frac{1}{3} \hat{\tau}+\hat{\tau}^{2}+\frac{5}{2} \hat{\tau}^{3}+8 \hat{\tau}^{4}+\frac{231}{8} \hat{\tau}^{5}+\cdots,
$$

where

$$
\hat{\eta} \equiv \frac{1}{3} \hat{R}_{\mathrm{P}} \sin ^{3} \theta_{\mathrm{P}} \sec ^{2} \theta_{\mathrm{P}} \eta,
$$


and

$$
\hat{\tau} \equiv \frac{1}{3} \exp (-\mathrm{i} \pi / 4) \hat{R}_{\mathrm{P}}^{2} \sin ^{5} \theta_{\mathrm{P}} \sec ^{4} \theta_{\mathrm{P}} \tau .
$$

The dependence of $\hat{r}$ on $\tau$ now follows from Eqs. (A2) and (A6).

According to Eqs. (63), (64), (744), (A1) and (A2)), the explicit form of the function $\left.F\right|_{C_{2}}$ is given by

$$
\begin{aligned}
\left.F\right|_{\xi=-\left(u_{S}{ }^{2}+\mathrm{i} \tau^{2}\right)^{1 / 2}} \simeq & \frac{1}{3} \hat{R}_{\mathrm{P}}^{-5} \csc ^{5} \theta_{\mathrm{P}} \mathbf{u}_{j}\left(\xi_{S}{ }^{2}+\mathrm{i} \tau^{2}\right)^{1 / 2} \\
& \times \exp \left[-\mathrm{i}\left(\mu \phi_{\mathrm{C}}-\pi / 2\right)\right] \partial\left(\eta^{-3}\right) /\left.\partial \tau\right|_{C_{2}}
\end{aligned}
$$

for $\hat{R}_{\mathrm{P}} \gg 1$. Insertion of this expression in Eq. (70) yields

$$
\begin{aligned}
\int_{C_{2}} \mathrm{~d} \xi F(\xi, \hat{z}) \exp \left(\mathrm{i} \alpha \xi^{2}\right)= & \frac{1}{3} \hat{R}_{\mathrm{P}}^{-8} \cot ^{6} \theta_{\mathrm{P}} \csc ^{5} \theta_{\mathrm{P}} \exp \left[-\mathrm{i}\left(\mu \phi_{S}+\pi / 4\right)\right] \\
& \times\left.\mathbf{u}_{j}\left(3 \mathcal{I}_{1}-\mathcal{I}_{2}\right)\right|_{C_{2}}
\end{aligned}
$$

in which

$$
\mathcal{I}_{1} \equiv \int_{0}^{\infty} \mathrm{d} \tau \tau^{-4} \exp \left(-\alpha \tau^{2}\right) \psi(\tau)
$$

and

$$
\mathcal{I}_{2} \equiv \int_{0}^{\infty} \mathrm{d} \tau \tau^{-3} \exp \left(-\alpha \tau^{2}\right)(\mathrm{d} \psi / \mathrm{d} \tau)
$$

with $\psi \equiv(\hat{\eta} / \hat{\tau})^{-3}$. Here, we have used the fact that $\mu \phi_{\mathrm{C}}-\alpha u_{S}{ }^{2}=\mu \phi_{\mathrm{S}}$, where $\phi_{\mathrm{S}}$ stands for the value of $\phi_{-}$at $\mathrm{S}$.

That the integrals $\mathcal{I}_{1}$ and $\mathcal{I}_{2}$ have turned out to diverge is a consequence of our having interchanged the orders of integration and differentiation in Eq. (37) [see also Eq. (13)]. The standard technique for regularizing such divergent integrals is to treat them as generalized functions whose physically significant values (i.e., the values that we would have found had we not interchanged the orders of integration and differentiation) are given by their Hadamard finite parts [19].

To apply the technique to $\mathcal{I}_{1}$, one begins by appealing to Taylor's Theorem to represent the continuously differentiable function $\psi$ as

$$
\psi(\tau)=\psi(0)+\psi^{\prime}(0) \tau+\frac{1}{2} \psi^{\prime \prime}(0) \tau^{2}+\frac{1}{3 !} \psi^{\prime \prime \prime}(0) \tau^{3}+\frac{1}{4 !} \psi^{\prime \prime \prime \prime}(\kappa \tau) \tau^{4},
$$

where $\kappa$ is a number lying between 0 and 1 . One then inserts Eq. (A11) in Eq. (A9) to 
rewrite $\mathcal{I}_{1}$ as

$$
\begin{aligned}
\mathcal{I}_{1}= & \lim _{\epsilon \rightarrow 0}\left[\psi(0) \int_{\epsilon}^{\infty} \mathrm{d} \tau \tau^{-4} \exp \left(-\alpha \tau^{2}\right)+\psi^{\prime}(0) \int_{\epsilon}^{\infty} \mathrm{d} \tau \tau^{-3} \exp \left(-\alpha \tau^{2}\right)\right. \\
& +\frac{1}{2} \psi^{\prime \prime}(0) \int_{\epsilon}^{\infty} \mathrm{d} \tau \tau^{-2} \exp \left(-\alpha \tau^{2}\right)+\frac{1}{3 !} \psi^{\prime \prime \prime}(0) \int_{\epsilon}^{\infty} \mathrm{d} \tau \tau^{-1} \exp \left(-\alpha \tau^{2}\right) \\
& \left.+\frac{1}{4 !} \int_{\epsilon}^{\infty} \mathrm{d} \tau \psi^{\prime \prime \prime \prime}(\kappa \tau) \exp \left(-\alpha \tau^{2}\right)\right] .
\end{aligned}
$$

The first four integrals inside the square brackets in this expression can be easily evaluated as functions of $(\alpha, \epsilon) ;$ e.g.,

$$
\int_{\epsilon}^{\infty} \mathrm{d} \tau \tau^{-4} \exp \left(-\alpha \tau^{2}\right)=\frac{1}{3} \epsilon^{-3}\left(1-2 \alpha \epsilon^{2}\right) \exp \left(-\alpha \epsilon^{2}\right)+\frac{2}{3} \pi^{1 / 2} \alpha^{3 / 2} \operatorname{erfc}\left(\alpha^{1 / 2} \epsilon\right),
$$

in which the error function $\operatorname{erfc}\left(\alpha^{1 / 2} \epsilon\right)$ approaches unity in the limit $\epsilon \rightarrow 0$.

The remaining fifth integral on the right-hand side of Eq. (A12) equals

$$
\begin{aligned}
\int_{\epsilon}^{\infty} \mathrm{d} \tau \psi^{\prime \prime \prime \prime}(\kappa \tau) \exp \left(-\alpha \tau^{2}\right)= & 4 ! \int_{\epsilon}^{\infty} \mathrm{d} \tau \tau^{-4}\left[\psi(\tau)-\psi(0)-\psi^{\prime}(0) \tau-\frac{1}{2} \psi^{\prime \prime}(0) \tau^{2}\right. \\
& \left.-\frac{1}{3 !} \psi^{\prime \prime \prime}(0) \tau^{3}\right] \exp \left(-\alpha \tau^{2}\right)
\end{aligned}
$$

by virtue of Eq. (A11). For $\alpha \gg 1$ (i.e., $\hat{R}_{\mathrm{P}} \gg 1$ ) and $\epsilon=0$, the leading term in the asymptotic value of the right-hand integral in Eq. (A14) is given by

$$
\int_{0}^{\infty} \mathrm{d} \tau \psi^{\prime \prime \prime \prime}(\kappa \tau) \exp \left(-\alpha \tau^{2}\right) \simeq \psi^{\prime \prime \prime \prime}(0) \int_{0}^{\infty} \mathrm{d} \tau \exp \left(-\alpha \tau^{2}\right)=\frac{1}{2}(\pi / \alpha)^{1 / 2} \psi^{\prime \prime \prime \prime}(0) .
$$

Here, we have applied l'Hôpital's rule to remove the indeterminacy in the value of the kernel of the right-hand integral in Eq. (14) at $\tau=0$.

Hadamard's finite part of the limiting version of each of the integrals that appear inside the square brackets in Eq. (A12) is obtained by simply discarding those terms in its representation as a function of $(\alpha, \epsilon)$ that diverge when $\epsilon$ tends to zero; e.g.,

$$
\mathcal{F}\left\{\int_{0}^{\infty} \mathrm{d} \tau \tau^{-4} \exp \left(-\alpha \tau^{2}\right)\right\}=\frac{2}{3} \pi^{1 / 2} \alpha^{3 / 2}
$$

according to Eq. (A13). Thus, Eq. (A15) and the finite parts of the divergent integrals on the right-hand side of Eq. (A12) jointly yield

$$
\begin{aligned}
\mathcal{F}\left\{\mathcal{I}_{1}\right\} \simeq & \frac{2}{3} \pi^{1 / 2} \psi(0) \alpha^{3 / 2}+\frac{1}{2} \psi^{\prime}(0)(\ln \alpha+\gamma) \alpha-\frac{1}{2} \pi^{1 / 2} \psi^{\prime \prime}(0) \alpha^{1 / 2} \\
& -\frac{1}{12} \psi^{\prime \prime \prime}(0)(\ln \alpha+\gamma)+\frac{1}{48} \pi^{1 / 2} \psi^{\prime \prime \prime \prime}(0) \alpha^{-1 / 2}, \quad \alpha \gg 1,
\end{aligned}
$$


where $\gamma=0.57721$ is Euler's constant. The same procedure, when applied to the integral defined in Eq. (A10), results in

$$
\begin{aligned}
\mathcal{F}\left\{\mathcal{I}_{2}\right\} \simeq & \frac{1}{2} \psi^{\prime}(0)(\ln \alpha+\gamma) \alpha-\pi^{1 / 2} \psi^{\prime \prime}(0) \alpha^{1 / 2}-\frac{1}{4} \psi^{\prime \prime \prime}(0)(\ln \alpha+\gamma) \\
& +\frac{1}{12} \pi^{1 / 2} \psi^{\prime \prime \prime \prime}(0) \alpha^{-1 / 2}, \quad \alpha \gg 1
\end{aligned}
$$

The required derivatives of $\psi$ at $\tau=0$ can be read off the following expansion of $(\hat{\eta} / \hat{\tau})^{-3}$ :

$$
\psi=1-3 \hat{\tau}-\frac{3}{2} \hat{\tau}^{2}-4 \hat{\tau}^{3}-\frac{105}{8} \hat{\tau}^{4}+\cdots,
$$

which follows from Eq. (A6a) [see also Eq. (A6c)].

Evaluating the right-hand sides of Eqs. (A17) and (A18) with the aid of Eqs. (A6c) and (A19), and inserting the resulting expressions in Eq. (A8), we finally arrive at

$$
\mathcal{F}\left\{3 \mathcal{I}_{1}-\mathcal{I}_{2}\right\}=-\left(105 / 6^{4}\right) \sin ^{18} \theta_{\mathrm{P}}\left|\sec \theta_{\mathrm{P}}\right|^{15} \hat{R}_{\mathrm{P}}^{15 / 2}, \quad \hat{R}_{\mathrm{P}} \gg 1
$$

and hence, at Eq. (77).

[1] A. V. Bessarab, A. A. Gorbunov, S. P. Martynenko, and N. A. Prudkoy, IEEE Trans. Plasma Sci. 32, 1400 (2004), ISSN 0093-3813.

[2] A. Ardavan, W. Hayes, J. Singleton, H. Ardavan, J. Fopma, and D. Halliday, J. Appl. Phys. 96, 7760 (2004), ISSN 0021-8979, corrected version of 96(8), 4614-4631.

[3] A. V. Bessarab, S. P. Martynenko, N. A. Prudkoi, A. V. Soldatov, and V. A. Terekhin, Radiation Physics and Chemistry 75, 825 (2006), ISSN 0969-806X.

[4] B. M. Bolotovskii and A. V. Serov, Radiation Physics and Chemistry 75, 813 (2006), ISSN 0969-806X.

[5] B. M. Bolotovskii and V. L. Ginzburg, Sov. Phys. Usp. 15, 184 (1972).

[6] V. L. Ginzburg, Sov. Phys. JETP 35, 92 (1972), ISSN 0038-5646.

[7] B. M. Bolotovskii and V. P. Bykov, Sov. Phys. Usp. 33, 477 (1990), ISSN 0038-5670.

[8] H. Ardavan, Phys. Rev. E 58, 6659 (1998), ISSN 1063-651X.

[9] H. Ardavan, A. Ardavan, and J. Singleton, J. Opt. Soc. Am. A 21, 858 (2004), ISSN 1084-7529.

[10] H. Ardavan, A. Ardavan, J. Singleton, J. Fasel, and A. Schmidt, J. Opt. Soc. Am. A 24, 2443 (2007). 
[11] The superposition of the subbeams is necessarily incoherent because the subbeams that are detected at two neighboring points within the overall beam arise from two distinct filamentary parts of the source with essentially no common elements. The incoherence of this superposition would ensure that, though the field amplitude within a subbeam, which narrows with distance, decays nonspherically, the field amplitude associated with the overall radiation beam, which occupies a constant solid angle, does not.

[12] J. D. Jackson, Classical Electrodynamics (Wiley, New York, 1999), 3rd ed.

[13] J. H. Hannay, Proc. Roy. Soc. A 452, 2351 (1996), ISSN 1364-5021.

[14] J. H. Hannay, Phys. Rev. E 62, 3008 (2000), ISSN 1063-651X.

[15] J. H. Hannay, J. Math. Phys. 42, 3973 (2001), ISSN 0022-2488.

[16] J. H. Hannay, J. Opt. Soc. Am. A 23, 1530 (2006), ISSN 1084-7529.

[17] H. Ardavan, A. Ardavan, and J. Singleton, J. Opt. Soc. Am. A 23, 1535 (2006), ISSN 10847529 .

[18] P. M. Morse and H. Feshbach, Methods of Theoretical Physics, vol. 1 (McGraw-Hill, New York, 1953).

[19] R. F. Hoskins, Delta Functions: An Introduction to Generalised Functions (Horwood, Chichester, 1999), chap. 7.

[20] C. Chester, B. Friedman, and F. Ursell, Proc. Camb. Phil. Soc. 53, 599 (1957).

[21] R. Burridge, SIAM J. Appl. Math. 55, 390 (1995), ISSN 0036-1399.

[22] N. Bleistein and R. A. Handelsman, Asymptotic Expansions of Integrals (Dover, New York, 1986).

[23] That these components of the gradient are of the same order of magnitude is a consequence of the fact that the spiraling cusps that emanate from this source propagate to infinity along a conical surface centered at the origin and so have nonzero pitch angles.

[24] M. V. Popov, V. A. Soglasnov, V. I. Kondrat'ev, S. V. Kostyuk, and Y. P. Ilyasov, Astron. Rep. 50, 55 (2006), ISSN 1063-7729.

[25] T. H. Hankins, J. S. Kern, J. C. Weatherall, and J. A. Eilek, Nature (London) 422, 141 (2003), ISSN 0028-0836.

[26] V. A. Soglasnov, M. V. Popov, N. Bartel, W. Cannon, A. Y. Novikov, V. I. Kondratiev, and V. I. Altunin, Astrophys. J. 616, 439 (2004), ISSN 0004-637X.

[27] A. Schmidt, H. Ardavan, J. Fasel, J. Singleton, and A. Ardavan, in Proceedings of the 363rd 
WE-Heraeus Seminar on Neutron Stars and Pulsars, edited by W. Becker and H. H. Huang (2007), pp. 124-127, arXiv:astro-ph/0701257. 